\title{
The long noncoding RNA NEAT1_1 is seemingly dispensable for normal tissue homeostasis and cancer cell growth
}

\author{
CARMEN ADRIAENS, ${ }^{1,2}$ FLORIAN RAMBOW, ${ }^{1,2}$ GREET BERVOETS, ${ }^{1,2}$ TOOMAS SILLA, $^{3}$ MARI MITO ${ }^{4}$ \\ TOMOKI CHIBA, ${ }^{5}$ HIROSHI ASAHARA, ${ }^{5}$ TETSURO HIROSE, ${ }^{6}$ SHINICHI NAKAGAWA, ${ }^{7}$ TORBEN \\ HEICK JENSEN, ${ }^{3}$ and JEAN-CHRISTOPHE MARINE ${ }^{1,2}$ \\ ${ }^{1}$ Laboratory for Molecular Cancer Biology, Center for Cancer Biology, VIB, 3000 Leuven, Belgium \\ ${ }^{2}$ Laboratory for Molecular Cancer Biology, Oncology Department, KU Leuven, 3000 Leuven, Belgium \\ ${ }^{3}$ Department of Molecular Biology and Genetics, Aarhus University, 8000 Aarhus, Denmark \\ ${ }^{4}$ RNA Systems Biochemistry Laboratory, RIKEN Cluster for Pioneering Research, 351-0198 Saitama, Japan \\ ${ }^{5}$ Department of Systems BioMedicine, Tokyo Medical and Dental University, 113-8510 Tokyo, Japan \\ ${ }^{6}$ Institute for Genetic Medicine, Hokkaido University, 060-0808 Sapporo, Japan \\ ${ }^{7}$ Faculty of Pharmaceutical Sciences, Hokkaido University, 060-0812 Sapporo, Japan
}

\begin{abstract}
NEAT1 is one of the most studied IncRNAs, in part because its silencing in mice causes defects in mammary gland development and corpus luteum formation and protects them from skin cancer development. Moreover, depleting NEAT1 in established cancer cell lines reduces growth and sensitizes cells to DNA damaging agents. However, NEAT1 produces two isoforms and because the short isoform, NEAT1_1, completely overlaps the $5^{\prime}$ part of the long NEAT1_2 isoform; the respective contributions of each of the isoforms to these phenotypes has remained unclear. Whereas NEAT1_1 is highly expressed in most tissues, NEAT1_2 is the central architectural component of paraspeckles, which are nuclear bodies that assemble in specific tissues and cells exposed to various forms of stress. Using dual RNA-FISH to detect both NEAT1_1 outside of the paraspeckles and NEAT1_2/NEAT1 inside this nuclear body, we report herein that NEAT1_1 levels are dynamically regulated during the cell cycle and targeted for degradation by the nuclear RNA exosome. Unexpectedly, however, cancer cells engineered to lack NEAT1_1, but not NEAT1_2, do not exhibit cell cycle defects. Moreover, Neat1_1-specific knockout mice do not exhibit the phenotypes observed in Neat1-deficient mice. We propose that NEAT1 functions are mainly, if not exclusively, attributable to NEAT1_2 and, by extension, to paraspeckles.
\end{abstract}

Keywords: IncRNA; NEAT1 isoforms; mouse genetics; cell cycle; RNA exosome; paraspeckles

\section{INTRODUCTION}

Long noncoding RNAs (IncRNAs) exceed 200 nucleotides (nt) in length and lack protein-coding potential. In the past decade, some of these molecules have arisen as prominent players in a range of cellular processes, including the formation of gene regulatory domains, the spatial organization of the genome, or cell plasticity (Quinn and Chang 2016). One of these IncRNAs, NEAT1, is required for the assembly of intriguing and enigmatic nuclear bodies known as paraspeckles (PSs) (Clemson et al. 2009; Sasaki et al. 2009; Sunwoo et al. 2009). Since then, PSs have been implicated in gene expression regulation and

Corresponding author: jeanchristophe.marine@kuleuven.vib.be Article is online at http://www.rnajournal.org/cgi/doi/10.1261/rna. 071456.119. in the maintenance of DNA integrity in response to endogenous and exogenous forms of stresses (Prasanth et al. 2005; Chen and Carmichael 2009; Choudhry et al. 2014; Hirose et al. 2014; Imamura et al. 2014; Adriaens et al. 2016; Mello et al. 2017; Ahmed et al. 2018; Lellahi et al. 2018). This may occur through their interaction with the RNA interference (RNAi) machinery and with micro-RNAs (Jiang et al. 2017; Li et al. 2018; Shuaib et al. 2019) or via the modulation of transcriptional and posttranscriptional regulators (Hirose et al. 2014; Hu et al. 2015; Kawaguchi et al. 2015; Torres et al. 2016; Morchikh et al. 2017;

(C) 2019 Adriaens et al. This article is distributed exclusively by the RNA Society for the first 12 months after the full-issue publication date (see http://rnajournal.cshlp.org/site/misc/terms.xhtml). After 12 months, it is available under a Creative Commons License (Attribution-NonCommercial 4.0 International), as described at http:// creativecommons.org/licenses/by-nc/4.0/. 
Hupalowska et al. 2018; Wang et al. 2018). Importantly, the generation of Neat1-deficient mice has highlighted a critical role for this IncRNA in the formation of a functional lactating mammary gland and corpus luteum (Nakagawa et al. 2014; Standaert et al. 2014; Adriaens et al. 2016). NEAT1 was also shown to protect preneoplastic cells from accumulating excessive DNA damage and, thereby, to be required for tumor initiation (Adriaens et al. 2016).

Because of the above, NEAT1 has emerged as one of the most studied IncRNAs. However, several outstanding questions remain regarding NEAT1 biology. The NEAT1 locus produces two different IncRNAs: NEAT1_2, a long transcript of $\sim 22.7 \mathrm{~kb}$, and NEAT1_1, a shorter transcript of $3.7 \mathrm{~kb}$ (Sasaki et al. 2009). One of the key unanswered questions to date is what the actual contributions of these two distinct isoforms are to the above-described phenotypes.

NEAT1_1 is a highly conserved and abundant polyadenylated transcript that is detected in virtually all tissues (Nakagawa et al. 2011). In contrast, expression of NEAT1_2, which is required for PS assembly, is only detected under specific physiological conditions (i.e., mammary gland development, corpus luteum formation) and in response to various forms of stress, including oncogenic stress. Conversely, nondifferentiated cells preferentially produce NEAT1_1, and consequently lack NEAT1_2 and thereby PSs (Nakagawa et al. 2011; Modic et al. 2019). Interestingly, PSs appear in $>65 \%$ of human epithelial cancers (Adriaens et al. 2016), where they predict poor prognosis (Li et al. 2018). In contrast, they are either completely absent or only sporadically detected in the adjacent normal tissues (Adriaens et al. 2016).

NEAT1_2 is a readthrough transcript that is produced as a result of incomplete processing of the 3 '-end of NEAT1_1. Little is known about the mechanisms that regulate NEAT1_13'-end processing, other than that it depends on the activity of a ubiquitous nucleic acid-binding protein, hnRNP $\mathrm{K}$, and the 3 '-end cleavage factor Im (CFIm) complex (Naganuma et al., 2012). PS assembly therefore depends on this poorly understood switch from transcriptional termination to readthrough (Naganuma et al. 2012; Yamazaki et al. 2018). Because PSs are detected in the cellular compartments that exhibit phenotypes following silencing of the Neat1 locus, it has been tempting to speculate that these defects arose as a consequence of loss of Neat1_2 and PSs. However, the investigated mice were also deficient for Neat1_1, and its contribution to these phenotypes has therefore remained unclear. The complete overlap of NEAT1_1 with the 5'-end of NEAT1_2 factors has made it particularly challenging to study the individual contribution and behavior of these two isoforms independently. As a result, most groups that study NEAT1 biology do not discriminate whether the observed effects in NEAT1 perturbation experiments are attributable to NEAT1_1, NEAT1_2, or both.
To study whether the two isoforms functionally interact as recently proposed (Fox et al. 2018) or whether they exert distinct biological functions, we used dual RNA-FISH, isoform-specific gene editing, and knockdown strategies. We show that the two isoforms are differentially expressed at various phases of the cell cycle and that NEAT1_1 is a target for degradation by the nuclear RNA exosome machinery. However, despite the high evolutionary conservation, the ubiquitous expression, and its tight regulation between the cell cycle, mice and cells deficient for NEAT1_1 did not exhibit any of the phenotypes observed upon ablation of both isoforms or NEAT1_2 only. Moreover, the phenotypes observed upon silencing NEAT1_2 in NEAT1_1-proficient cells were recapitulated in NEAT1_1-deficient cells. We propose that NEAT1's biological functions are solely attributable to the NEAT1_2 isoform and by extension to PS formation. The pathophysiological function of NEAT1_1, if any, remains to be elucidated. Our study, therefore, encourages a more careful dissection of individual noncoding RNA isoforms and indicates that high abundance and conservation is not necessarily predictive of functionality.

\section{RESULTS}

\section{Differential regulation of NEAT1 isoforms in response to stress}

To dissect a putative differential behavior of the two NEAT1 isoforms in cultured cancer cells, we performed RNA-FISH with two distinct probes that target both transcripts (red) or NEAT1_2 specifically (blue) (Fig. 1A). As the first portion of NEAT1_2 completely overlaps the short isoform, a pink signal (red + blue) marks the presence of both transcripts, whereas red signals indicate the sole presence of NEAT1_1 outside of PSs. Note that this approach does not allow us to determine whether NEAT1_1 localizes to PSs (Clemson et al. 2009; Souquere et al. 2010). Using this method, we observed a fraction of untreated, proliferating U2OS cells displaying NEAT1_1 in the nucleoplasm, outside of PS (37.7 $\pm 15.8 \%$ of the cells) (Fig. 1B,C, left panel and box plot). U2OS cells are triploid for NEAT1 and, consistently, often three pink dots were detectable, indicating PS formation at those loci (Clemson et al. 2009; Mao et al. 2011). We and others have shown that induction of p53 stimulates transcription of NEAT1 and PS formation (Blume et al. 2015; Adriaens et al. 2016; Idogawa et al. 2017; Mello et al. 2017). Accordingly, treatment of the cells with the p53 inducer Nutlin-3a increased the size of PSs. This was accompanied by a dramatic increase in the proportion of cells displaying nucleoplasmic NEAT1_1-specific signal $(79.0 \pm 8.3 \%$ of the cells; Fig. 1B,C center panel and box plot). In contrast, exposure to the DNA-damaging agent hydroxyurea $(\mathrm{HU})$ decreased the NEAT1_1-specific 
A

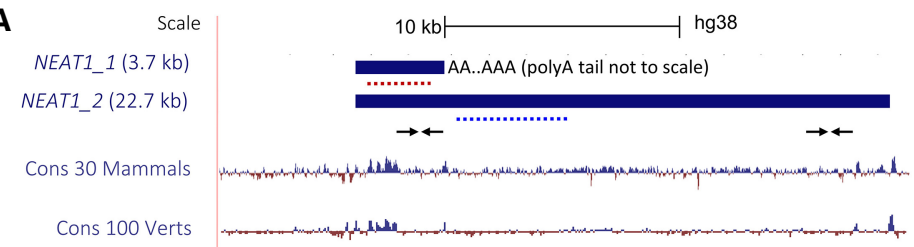

RepeatMasker | | | | | | | | || || ||| | || ||||||| | || ||

chr11: $\quad|65,425,000| 65,430,000|65,435,000| 65,440,000|65,445,000|$

B
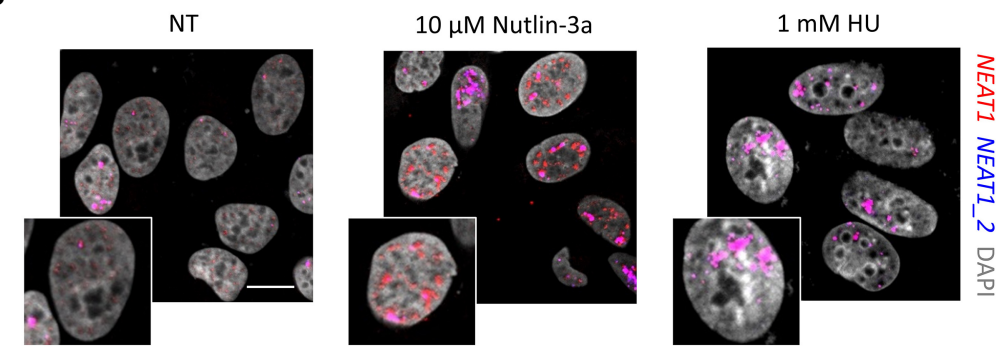

C

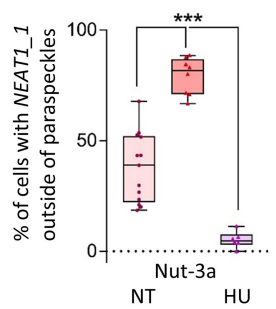

D

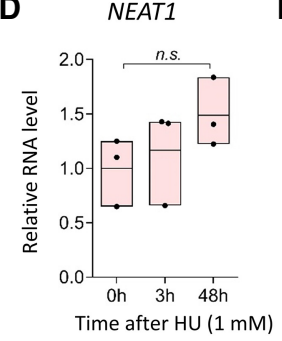

E

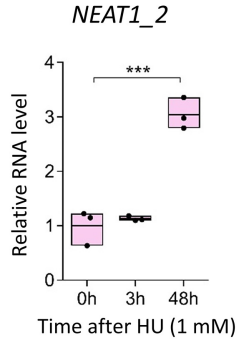

FIGURE 1. NEAT1 isoforms are differentially regulated by distinct p53 activating agents. (A) RefSeq representation of human NEAT1 isoforms in the UCSC genome browser (hg38), nucleotide-level conservation among 30 mammals and 100 vertebrates, and the location of repeats in the genome sequence. Red and blue dotted lines represent RNA-FISH probes targeting both and the long NEAT1_2 isoform specifically, respectively. Note that when targeting the long isoform, blue and red probes will overlap and thus show PSs in pink. Small arrows represent approximate locations of RT-qPCR primers used in this study. The poly(A) tail on the short NEAT1_1 isoform is not drawn to scale. (B) Representative confocal images of RNA-FISH targeting NEAT1 isoforms in U2OS cells in nontreated (NT), $10 \mu \mathrm{M}$ Nutlin-3a (24 h) and $1 \mathrm{mM}$ hydroxyurea (HU, $48 \mathrm{~h}$ ) conditions. Scale bar, $15 \mu \mathrm{m}$. (C) Quantification of the percentage of cells in which the short isoform can be observed outside of PSs. Each dot represents an independent experiment ( $N=13,8$, and 6, respectively). (D-F) Relative levels of NEAT1 (both isoforms; $D), N E A T 1$ 2 $(E)$, and the canonical p53 target CDKN1A $(F)$ after 0,3 , and $48 \mathrm{~h}$ of $\mathrm{HU}$ treatment $(1 \mathrm{mM})$ in RT-qPCR.

signal (with only $5.2 \pm 3.7 \%$ of the cells being positive; Fig. 1B,C, right panel and box plot).

Real-time quantitative PCR (RT-qPCR) analyses with primers detecting both isoforms and NEAT1_2 only (Fig. 1A for primer locations) established that NEAT1_2 was specifically up-regulated in cells exposed to HU (Fig. $1 D, E)$. As expected, an increase in the levels of the p53-target CDKN1A was also observed, indicating its transcriptional activation (Fig. 1F). Although we noted that the sizes of NEAT1_2-containing bodies slightly decreased in these cells, we confirmed that they were genuine PSs by costaining with NONO, a canonical PS marker (Supplemental Fig. S1; Fox et al. 2005; Souquere et al. 2010).

These data indicated that the ratio and localization of NEAT1_1 and NEAT1_2 vary depending on the type of

F

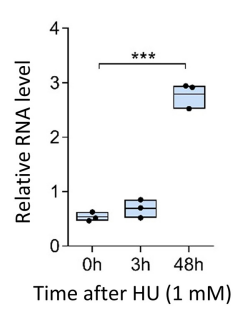

stress inflicted to the cells. The experiments also highlighted the presence of a large pool of NEAT1_1 that does not overlap with NEAT1_2containing PSs (Nakagawa et al. 2011; Li et al. 2017).

\section{NEAT1_1 levels are dynamically regulated during the cell cycle}

In the p53-competent U2OS cells, Nutlin-3a induces primarily a $G_{1}$ cell cycle block through activation of CDKN1A (Shen et al. 2008). In contrast, $\mathrm{HU}$ arrests cells in $\mathrm{S}$ phase through the inhibition of the deoxynucleotide (dNTP) producing enzyme ribonucleotide reductase (RNR), thereby depleting the dNTP pool during replication (Singh and Xu 2016). We therefore hypothesized that NEAT1_1 and NEAT1_2 levels may be differentially regulated during the cell cycle. To test this, we deprived cells from serum to halt them in a resting, $G_{0}$-like state $\left(G_{0}\right)$. Subsequently, cells were released in $20 \%$ serum in the presence of the DNA polymerase inhibitor aphidicolin to synchronize them at the $G_{1} / S$ phase boundary. DNA content analysis using flow cytometry confirmed efficient synchronization of cells (Fig. 2A). Using the above described dual RNA-FISH strategy, we observed that $87 \pm 15 \%$ of $\mathrm{G}_{0}$-halted cells expressed the short NEAT1_1 isoform outside of PSs (Fig. 2B,C; Supplemental Fig. S2A). In contrast, only $4.7 \pm 7.8 \%$ of the $\mathrm{G}_{1} / \mathrm{S}$-arrested cells displayed NEAT1_1-specific signals that did not overlap with NEAT1_2 (Fig. 2B,C; Supplemental Figs. S2A, S3A). RT-qPCR analysis demonstrated that total levels of NEAT1, but not of NEAT1_2, were increased in $\mathrm{G}_{0}$ cells (Supplemental Fig. S3C). Contrastingly, in $\mathrm{G}_{1} / \mathrm{S}$ cells, NEAT1 and NEAT1_2 levels were comparable and significantly lower as compared to nonsynchronized cells $(P=0.0018$ for NEAT1 and $P=$ 0.0077 for NEAT1_2, unpaired two-sided t-test using Holm-Sidak method) (Supplemental Fig. S3D). Whereas $\mathrm{G}_{0}$ cells displayed on average three NEAT1_2-containing PSs and $24 \pm 23$ NEAT1_1 RNA-FISH signals, respectively (Supplemental Fig. S2B,D), $\mathrm{G}_{1} / \mathrm{S}$ cells displayed on average 4.5 PSs per cell (Supplemental Fig. S2C). Together, these data indicated that, in nonproliferating cells, 
A

B
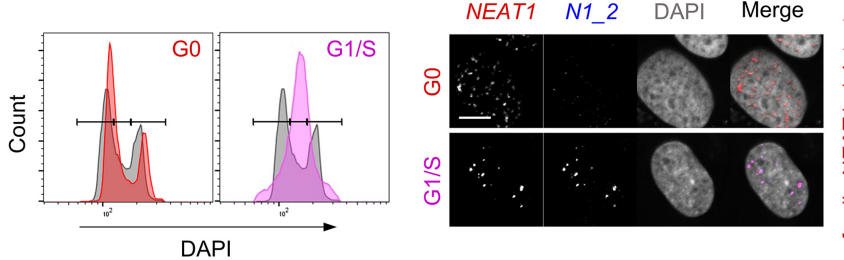

D

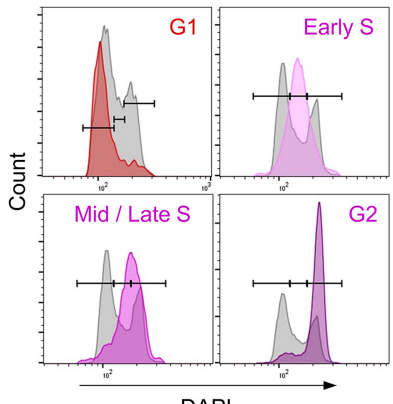

DAPI

G

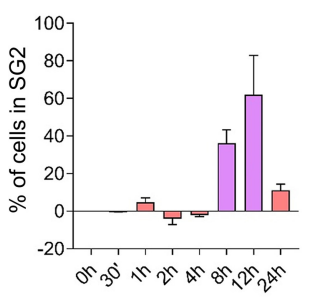

H

E NEAT1 N1 2 DAPI Merge
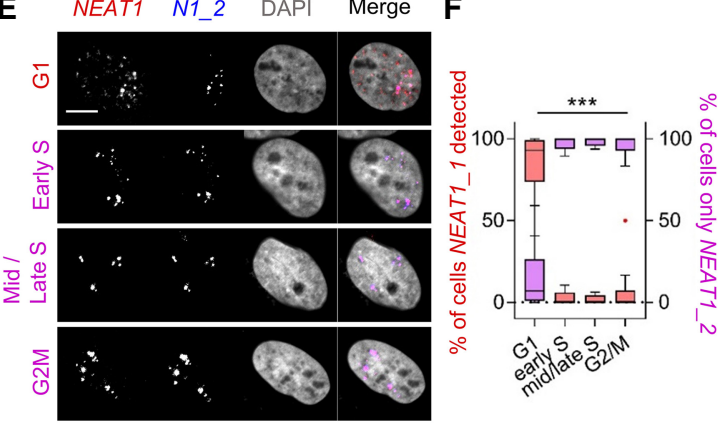

$\mathbf{J}$

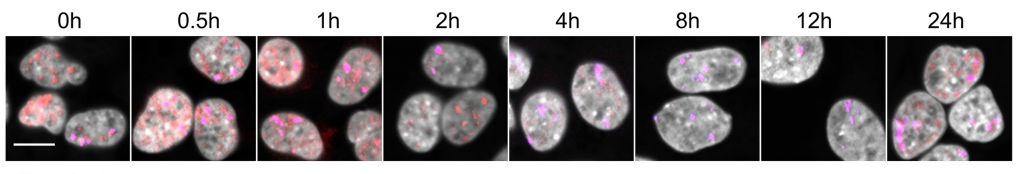

NEAT1 NEAT1_2 DAPI

FIGURE 2. NEAT1 isoforms are differentially regulated during the cell cycle. (A) Representative DNA content (DAPI) distribution of U2OS cells in $\mathrm{G}_{0}$ ( 3 d starvation) and $\mathrm{G}_{1} /$ $\mathrm{S}\left(\mathrm{G}_{0}\right.$ cells released in $20 \%$ serum $+5 \mu \mathrm{g} / \mathrm{mL}$ aphidicolin for $\left.24 \mathrm{~h}\right)$. Gray background plots are control nonsynchronized (NS) cells in culture. (B) Representative images of NEAT1/ NEAT1_2 RNA FISH of the cells in A. Scale bar, $10 \mu \mathrm{m}$. (C) Quantification of the percentage of cells in which NEAT1_1 was detected independently of NEAT1_2 PSs (red boxplots, left $y$-axis). Percentage of cells in which only NEAT1_2 PSs were detected (right $y$-axis, purple boxplots). Tukey plots of individual data points (one point per picture). Significance was calculated using an unpaired, two-sided t-test on independent biological replicates ( $N=$ at least three). $\left(^{* * *}\right) P<0.001$. (D) Like in $A$, but from U2OS cells synchronized by double thymidine block and released for $18\left(\mathrm{G}_{1}\right), 2$ (early S), 4 (mid/late S), and $8 \mathrm{~h}(\mathrm{G} 2 \mathrm{M})$. (E) Representative images of NEAT1/NEAT1_2 RNA FISH of the cells in D. (F) Same as $C$ for the cells in $D$. Significance was calculated using a two-way ANOVA with Dunnett's correction for multiple comparisons on independent replicates ( $N=$ at least three). (***) $P<0.001$ for $G_{1}$ vs. early $S$, $\mathrm{mid} /$ late $\mathrm{S}$, and $\mathrm{G}_{2}$. N1_2=NEAT1_2. (G) Percentage of cells in $\mathrm{S}$ and $\mathrm{G}_{2}$ phases at different time points relative to time $=0 \mathrm{~h}$ in HeLa cells upon release into $20 \%$ serum after $3 \mathrm{~d}$ starvation. $(H)$ Box plots (Min to Max) of individual data points ( $N=5$ and 3 pictures per replicate, respectively) quantifying the percentage of cells in E in which NEAT1_1 was detected using RNA-FISH against both and the long isoform specifically. (I) Relative increase of NEAT1_2 by RT-qPCR of the cells in $H$. Error bars are standard deviation of $N=2$ experiments in $G$ and $H$. $(J)$ Representative pictures of cells in G-I. Scale bar, $20 \mu \mathrm{m}$.

NEAT1_1 is the predominant isoform, and that, upon entering the cell cycle, the amount of signal for NEAT1_1 outside of PSs abruptly drops.
To substantiate these data, we used a conventional double thymidine block-and-release protocol to synchronize cells and subsequently release them for 2 (early S), 4-6 (mid/late $\mathrm{S}), 8\left(\mathrm{G}_{2} \mathrm{M}\right)$, or $17-20 \mathrm{~h}\left(\mathrm{G}_{1}\right)$ (Fig. 2D; Supplemental Fig. S3B). The majority of the cells in $\mathrm{G}_{1}(85.3 \pm$ 16.7\%) displayed detectable NEAT1_1 signal outside of PS, whereas in the early $\mathrm{S}$, mid/late $\mathrm{S}$, and $\mathrm{G}_{2} \mathrm{M}$ phases, NEAT1_1 signal was found in only a very small fraction of cells $(2.4 \pm$ $3.87 \% ; 1.63 \pm 2.6 \%$, and $5.8 \pm 13.1 \%$, respectively; Fig. 2E,F; Supplemental Fig. S2A). Only $2.2 \pm 1.4 \%$ of the cells did not display any detectable NEAT1 staining (data not shown). We next characterized the numbers of NEAT1 and NEAT1_2 signals per cell in $G_{1}$, $S$, and $G_{2}$ phases. In $G_{1}$ cells, the number of NEAT1_1 foci per cell varied greatly, with an average of $24.7 \pm 22$ (Supplemental Fig. S2D). In contrast, the number of NEAT1_2 detectable signals/PSs fluctuated between 6 and 8.5 (Supplemental Fig. S2B-D).

RT-qPCR analysis showed elevated levels of total NEAT1 in $\mathrm{G}_{1}$ compared to the other phases of the cell cycle. Because NEAT1_2 levels remained relatively constant throughout, this is a consequence of higher NEAT1_1 levels in this particular phase (Supplemental Fig. S3E-G). Accordingly, the ratio of the levels of NEAT1_2 over NEAT1 (NEAT1_1+ NEAT1_2) (Supplemental Fig. S3H) in early $\mathrm{S}$, mid/late $S$, and $\mathrm{G}_{2}$ phases revolved around 1 (mean $=\sim 1.4$ in early $\mathrm{S}$ and $\sim 0.98$ in both $\mathrm{mid} / \mathrm{late} \mathrm{S}$ and $\mathrm{G}_{2}$ ). In contrast, this ratio was consistently $<1$ (mean $=\sim 0.18$ ) in $\mathrm{G}_{1}$ cells, indicating that NEAT1_1 contributes to the total levels of NEAT1 in these cells. Moreover, in $G_{1} / G_{0}$ cells, no linear relationship could be established between NEAT1_2 and NEAT1 $\left(R^{2}=\right.$ 0.1141, P-value 0.259), whereas a significant positive correlation $\left(R^{2}=\right.$ $0.6488, P<0.001$ ) was observed in $S$ and $G_{2}$ cells. The $b 0$ and $b 1$ values of the equation for the linear regression (NEAT1 $=b_{0}+b_{1} \times$ NEAT1_2) were nearly 0 and 1 , respectively $\left(b_{0}=0.09, b_{1}\right.$ $=1.3$ ). These results strongly indicate that NEAT1_1 levels 
drop as cells engage in a new round of cell division. Similar results were obtained with another cancer cell line (HeLa cells; Fig. 2E-H). We therefore concluded that NEAT1_1 levels fluctuate during the cell cycle, whereas NEAT1_2 levels remain relatively constant. Because NEAT1_2 is the product of a transcription readthrough event, the down-regulation of NEAT1_1 as cells engaged in DNA replication cannot be due to a decrease in transcription, but must instead occur through active degradation of the transcript.

\section{NEAT1_1 is degraded by the RNA exosome}

To identify factors that contribute to the degradation of NEAT1_1, we mined publicly available data sets and observed that NEAT1_1, but not NEAT1_2, levels were up-regulated upon depletion of the exosome component RRP40 (Supplemental Fig. S4A). We confirmed these results by RNA-FISH (Fig. 3A,B) and RT-qPCR analysis. In RRP40 KD cells, we observed a lower ratio of NEAT1_2/ NEAT1 RNA-FISH signal per nucleus and a specific increase of the total levels of NEAT1, but not NEAT1_2, indicating that NEAT1_1 is specifically targeted by the RNA exosome machinery (Fig. 3C). Depletion of RRP40 results in the stabilization of a series of nuclear-polyadenylated RNAs, which accumulate in distinct poly $(A)^{+}$foci (Silla et al. 2018). Combining RNA-FISH probes targeting NEAT1 and poly $(A)^{+}$RNA, we detected an accumulation of NEAT1 in the poly $(\mathrm{A})^{+}$-rich foci (Fig. 3D,E; Supplemental Fig. S4B,C). We also noted that a pool of
A

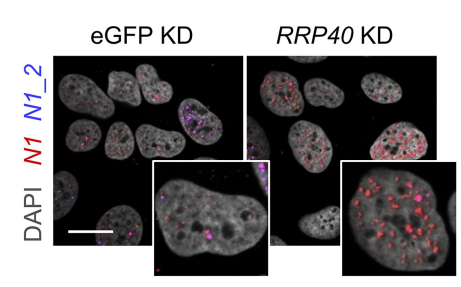

D

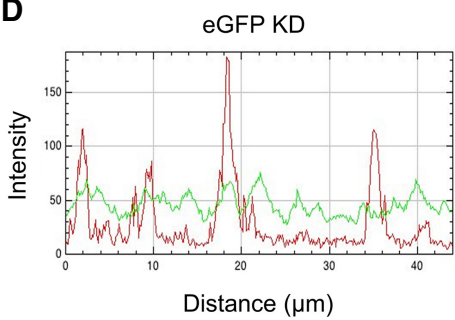

G

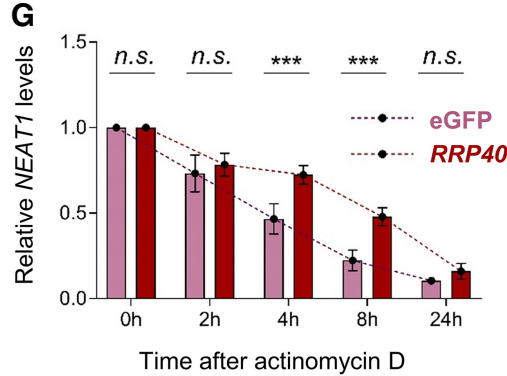

B

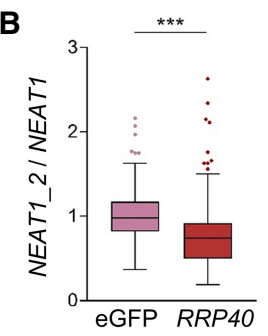

E

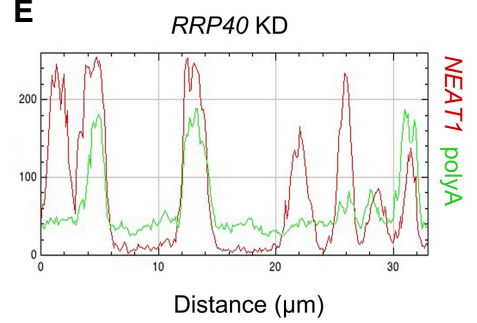

C

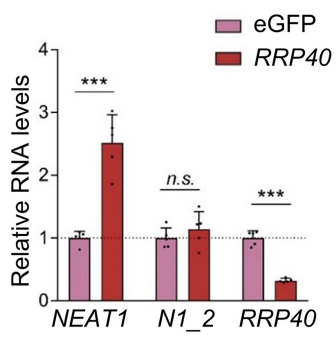

F NEAT1 PolyA NONO

H

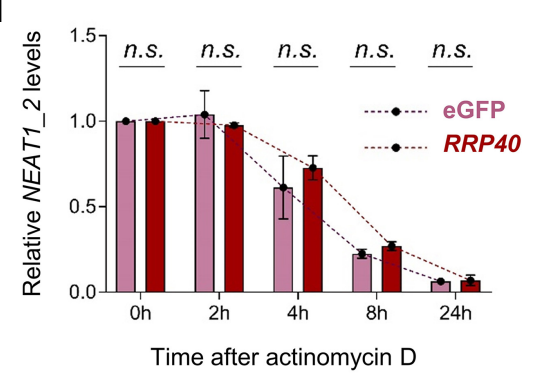

FIGURE 3. NEAT1_1 is selectively degraded by the RNA exosome. (A) Representative NEAT1/NEAT1_2 RNA-FISH images of U2OS cells in which RRP40, a subunit of the RNA exosome complex, was knocked down. (B) Tukey plots of quantified RNA-FISH signal shown as NEAT1_2 nuclear intensity over total NEAT1 nuclear intensity in $N=5$ independent experiments. Significance was calculated by an unpaired MannWhitney $U$ nonparametric test with each cell as a data point combining data from five independent experiments. $\left({ }^{* * *}\right)$ Two-tailed $P<0.001$. (C) Mean and standard deviation of relative RNA levels by RT-qPCR of the same experiments as in $B(N=5)$ in eGFP and RRP40 KD cells suggesting specific up-regulation of the NEAT1_1 isoform. Dots are individual data points. $(D, E)$ Line plots of the intensity in eGFP KD (D) and RRP40KD $(E)$ conditions showing NEAT1 localization in poly(A) ${ }^{+}$accumulations upon exosome inhibition. (F) NEAT1 (blue)/poly(A) (red) RNA-FISH and IF in HeLa cells showing NEAT1/poly(A) ${ }^{+}$accumulations in RRP40 KD conditions (arrows) are distinct from PSs (NEAT1 + NONO, asterisk). (G-l) Relative RNA levels of both NEAT1 isoforms $(G)$ and the long NEAT1_2 isoform specifically $(H)$ upon addition of $2 \mathrm{mg} / \mathrm{mL}$ of the transcription inhibitor actinomycin D $48 \mathrm{~h}$ after eGFP or RRP40 KD in HeLa cells. (I) Bar graph showing relative RNA levels of RRP40 for the experiments in $\mathrm{G}-\mathrm{H}$ at time $=\mathrm{O}$ h. Statistical significance was tested using a two-way ANOVA with Dunnett's correction for multiple testing for $N=3$ independent experiments. Bars and error are mean and standard deviation. Individual data points are independent experiments. 
NEAT1 did not overlap with poly $(A)^{+}$RNA, which likely represents NEAT1_2 RNAs, as these are not polyadenylated and rather stable throughout the cell cycle. To confirm this, we performed NEAT1- and poly $(\mathrm{A})^{+}-\mathrm{FISH}$ combined with immunofluorescence analysis of the canonical PS protein NONO. Consistent with our prediction and the absence of costaining with another PS marker, SFPQ (Silla et al. 2018), PSs, and NEAT1/poly(A) ${ }^{+}$foci did not overlap (Fig. 3F).

To confirm that the observed up-regulation of NEAT1_1 was due to a decrease in RNA degradation rather than a transcriptional effect, we measured NEAT1 and NEAT1_2 levels by RT-qPCR in RRP40-depleted cells at different time points following exposure to the transcriptional inhibitors actinomycin $D$ and $\alpha$-amanitin. The pace at which NEAT1_1, but not NEAT1_2, decays was significantly slower in RRP40 KD cells as compared to control cells (Fig. 3H-I; Supplemental Fig. S4D-F). We concluded that NEAT1_1 is specifically degraded by the RNA exosome.

\section{NEAT1_1 does not contribute to cell growth}

To investigate whether NEAT1_1 might play a role as a regulator of cell cycle progression and/or survival of $\mathrm{G}_{1}$ cells, we used CRISPR editing to delete a small regulatory region ( 140 bp) at the 3 '-end of NEAT1_1 spanning the CFIm and hnRNP K binding sites as well as the polyadenylation signal (PAS). This approach is expected to selectively delete NEAT1_1 by allowing transcription readthrough and constitutive NEAT1_2 expression. We introduced the deletion into U2OS and two other cancer cell lines, HCT116 p53 WT and its isogenic p53 KO line (Fig. 4A; Supplemental Fig. $\mathrm{S} 5 \mathrm{~A}, \mathrm{~B})$, and subsequently isolated single-cell clones. PCRbased genotyping confirmed successful homozygous targeting of the NEAT1_1 regulatory region, resulting in two wild-type (WT) and four NEAT1_1 knockout (KO) U2OS clones (Fig. 4B), as well as four WT and four KO clones for each of the HCT116 cell lines (Supplemental Fig. S5A,B).

To establish that the engineered cells did not express NEAT1_1, we quantified total NEAT1 and NEAT1_2 levels using RT-qPCR. Whereas relative NEAT1 levels did not change in the PAS KO clones, NEAT1_2 levels were increased, consistent with the prediction that all initiated transcripts contribute to the expression of the long isoform (Fig. 4C; Supplemental Fig. S5E,F). In agreement, RNAFISH analysis did not detect NEAT1_1 in the nucleoplasm of the KO clones (Fig. 4D; Supplemental Fig. S6C,D). Notably, PS integrity was preserved in the $\mathrm{KO}$ cells, as evidenced by their costaining with the PS marker NONO and the NEAT1 RNA-FISH probe sets (Supplemental Fig. S6).

We next assessed whether the NEAT1_1 deletion affects cell growth and proliferation. Long-term growth assays indicated that NEAT1_1 $\mathrm{KO}$ cells proliferated at a similar rate as the WT controls (Fig. 4E; Supplemental Fig. S5J-M), which was confirmed in a short-term growth assay (WST1) and following exposure to Nutlin-3a or a low dose of the DNA damaging agent doxorubicin (Fig. 4F; Supplemental Fig. S5G-I). This is in contrast to specific transient depletion of NEAT1_2, which sensitized cells to these agents (Adriaens et al. 2016). These results thus indicated that NEAT1_1 is not required for the two-dimensional growth and proliferation of cancer cell lines.

To further assess NEAT1_1- and NEAT1_2-independent functions, we knocked down NEAT1_2 in the PAS KO cells and analyzed their cell cycle distribution and growth properties. NEAT1_2 knockdown induced a similar decrease in EdU-positive cells as it did in WT, cycling cells (Fig. 4G,H). Cell density was also markedly decreased upon NEAT1_2 KD (Fig. 4I). These data indicated that NEAT1_1 does not contribute to the ability of NEAT1_2 to preserve the genomic integrity of cancer cell lines.

Moreover, we could not identify cell cycle defects in RRP40-depleted cells, indicating that NEAT1_1 accumulation in poly $(A)^{+}$foci does not affect cell division (Fig. 4J,K).

\section{NEAT1_1 depletion does not overtly impact on the cellular transcriptome}

It has been proposed that NEAT1 regulates cellular gene expression by localizing to the transcription start sites of actively transcribed genes (West et al. 2014). To test whether NEAT1_1, which is found prominently in the nucleoplasm of $\mathrm{G}_{0}$ and $\mathrm{G}_{1} / \mathrm{S}$ cells (Fig. 5A), modulates transcription, we profiled the transcriptome of PAS $\mathrm{KO}$ and WT ctrl cells by RNA-seq. We detected on average $18.030\left(G_{0}\right)$ and $17.250\left(G_{1} S\right)$ expressed genes (Fig. 5B), of which only $156(\sim 0.86 \%)$ and $23(\sim 0.13 \%)$, respectively, were significantly differentially expressed (DE) in the PAS KO compared to WT cells (Fig. 5C-F). Gene ontology analysis did not identify particular pathways or biological processes affected by the depletion of NEAT1_1. Thus, although NEAT1_1 is highly expressed in $\mathrm{G}_{0} / \mathrm{G}_{1}$ cells, its loss does not significantly impact the overall gene expression profiles of these cells.

\section{Neat1_1 KO mice do not exhibit lactation nor fertility defects}

To further explore a physiological function of Neat1_1 in normal cells and in the relevant in vivo context, we generated a Neat1_1-specific KO mouse strain using a strategy similar to the one used to knock out NEAT1_1 in cells. In brief, 39 base pairs surrounding the PAS of Neat1_1 were excised using CRISPR/Cas9 in mouse embryonic stem cells (mESCs), generating a mouse strain deficient for Neat1_1 (Isobe et al. 2019). PAS KO mice were born at the expected Mendelian ratios (Fig. 6D) and did not exhibit the lactation defect previously observed in Neat1 full KO mice (Standaert et al. 2014). We weighed pups born in 
A

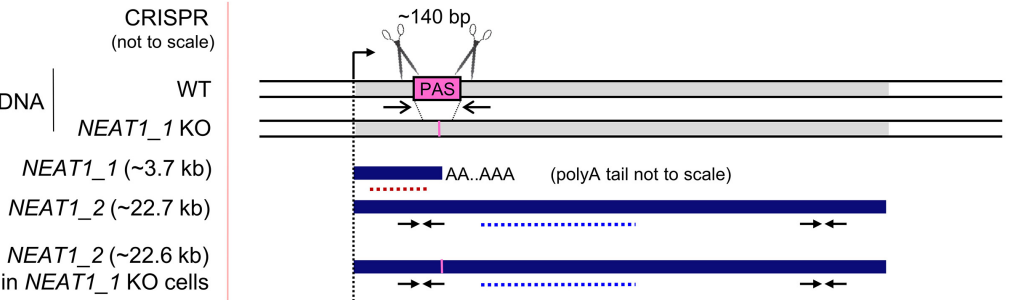

B in NEAT̄_1 $1 \mathrm{KO}$ cells
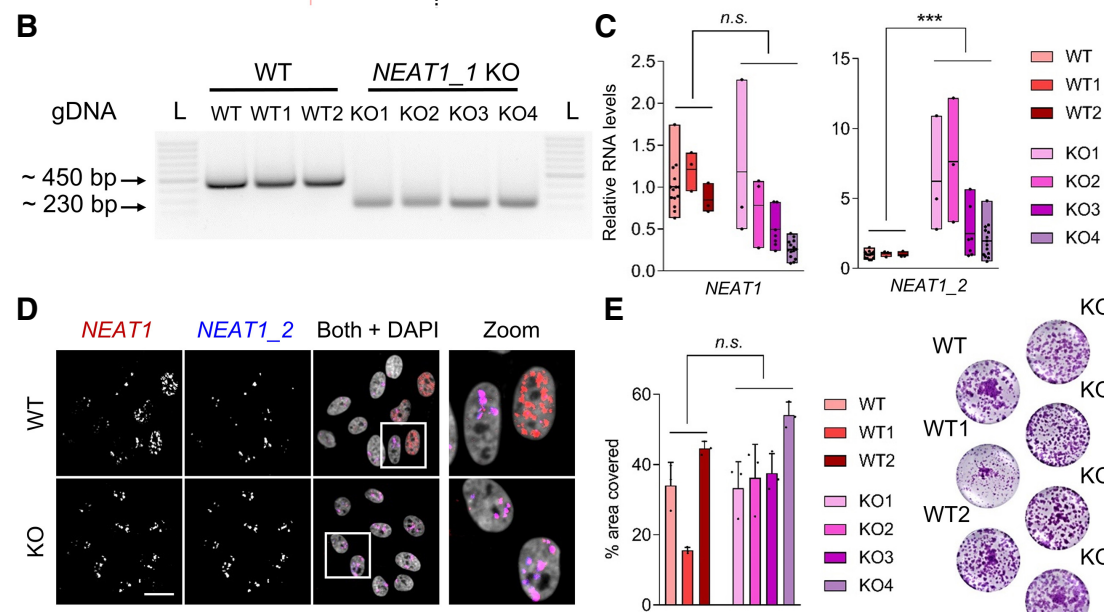

$\mathbf{E}$
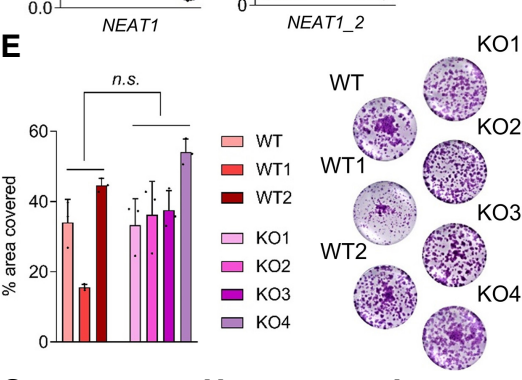

$\mathbf{F}$
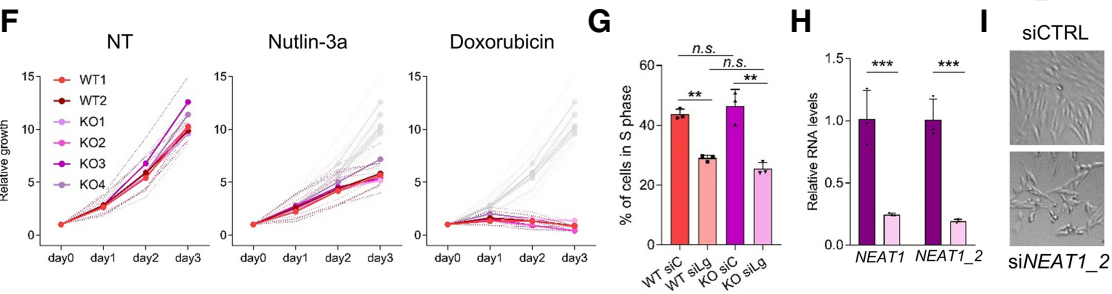

$\mathbf{J}$

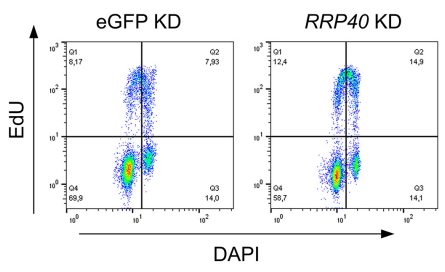

$\mathbf{K}$

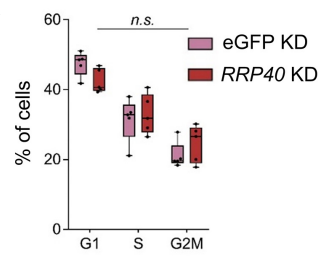

FIGURE 4. Loss of NEAT1_1 does not impact on cell growth. (A) Scheme of the CRISPR strategy used to knock out NEAT1_1 by deletion of the regulatory sequences and PAS at the $3^{\prime}$-end of the NEAT1_1 short isoform genomic sequence and the resulting RNAs in wild-type (WT) and NEAT1_1 knockout $(\mathrm{KO})$ cells. Arrows and dotted lines in the RNA represent RT-PCR primers and RNA-FISH probes, respectively. Arrows on the DNA sequence represent approximate locations of genotyping primers. (B) Representative inverted gel image of the PCR product from U2OS gDNA used for genotyping individually isolated single cell clones after CRISPR with the primers depicted in $A$. (L) DNA ladder. (C) Relative RNA levels of NEAT1 (total) and NEAT1_2 specifically showing up-regulation of NEAT1_2, whereas the total levels of NEAT1 remain the same in WT versus KO clones. The WT without a number is the mother population from which the WT and KO clones were derived. Significance was calculated using a two-way ANOVA comparing RNA levels in WT and KO clones with Dunnett's correction for multiple testing. $\left(^{* * *}\right) P<0.001$ for $N=$ at least three independent experiments. (n.s.) Not significant. Dots represent data points from each independent experiment. (D) Representative NEAT1/NEAT1_2 RNA-FISH image of WT and NEAT1_1 KO cells showing the complete loss of NEAT1_1 upon poly(A) site knockout. Scale bar, $10 \mu \mathrm{m}$. (E) Quantification of percentage of area covered (left) and representative images (right) of colony assays $14 \mathrm{~d}$ after seeding 2000 cells per well in $N=3$ independent replicates, three wells per replicate each. Statistical testing was done using a one-way ANOVA. (n.s.) Not significant. Dots represent the average of three wells of the independent experiments. Bars are mean + standard deviation. $(F)$ Shortterm growth measured by WST-1 relative to day 0 (1 d after seeding) in nontreated (NT), $10 \mu \mathrm{M}$ Nutlin-3a and a low dose of doxorubicin (150 ng/ $\mathrm{mL}$ ) in NEAT1_1 WT and KO clones. All data is the average of $N=3$ independent experiments. Standard error is depicted as dotted lines above and below the data points. In Nutlin-3a and doxorubicin conditions, values in nontreated conditions are shown as light gray lines in the back of the graph. All data is not significant as tested by two-way ANOVA with Dunnett's correction for multiple testing in the different time points. (G) Quantification of percentage of EdU-positive cells (S phase) in flow cytometry upon CTRL (siC) and NEAT1_2 (siLg) knockdown in WT and KO clones. $N=3$ independent experiments. Significance was determined using two-way ANOVA with Dunnett's correction for multiple comparisons. $(* * *) P<0.001$. (n.s.) Not significant. (H) NEAT1_2 knockdown efficiency as assessed by RT-qPCR $48 \mathrm{~h}$ after transfection for siCTRL (dark purple bars) and siNEAT1_2 (light purple bars) for the NEAT1_1 KO cells shown in the pictures on the right (panel I). (I) Representative image of siCTRL and siNEAT1_2 KO cells showing decreased cell density $48 \mathrm{~h}$ after transfection. $(\mathrm{J})$ Representative flow cytometry graphs of EdU/DAPI staining in eGFP and RRP40 KD conditions. (K) Percentage of cells in $\mathrm{G}_{1}, \mathrm{~S}_{\text {, and }} \mathrm{G}_{2} \mathrm{M}$ phases of the cell cycle upon eGFP and RRP40 KD as in Figure 3A-C. $N=5$ independent experiments. Nonsignificance was determined using a two-way ANOVA comparing eGFP and RRP40 conditions in each of the phases with Dunnett's correction for multiple testing. 
A

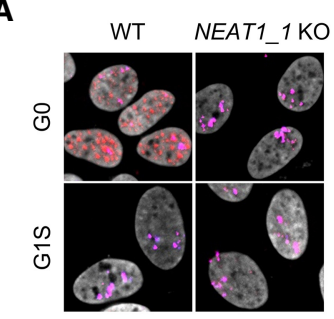

C

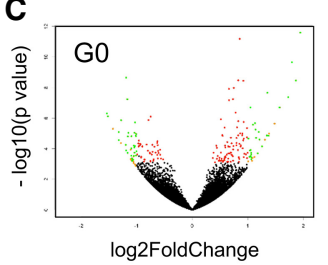

$\mathbf{F}$

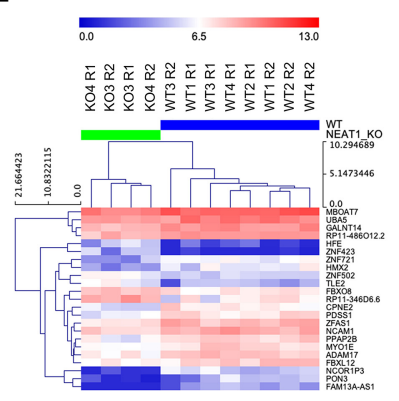

B

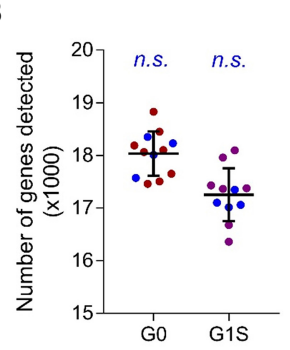

D

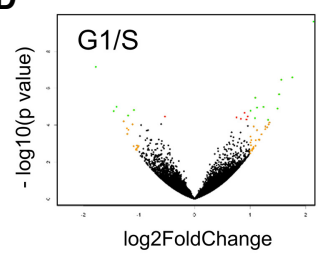

$\mathbf{E}$

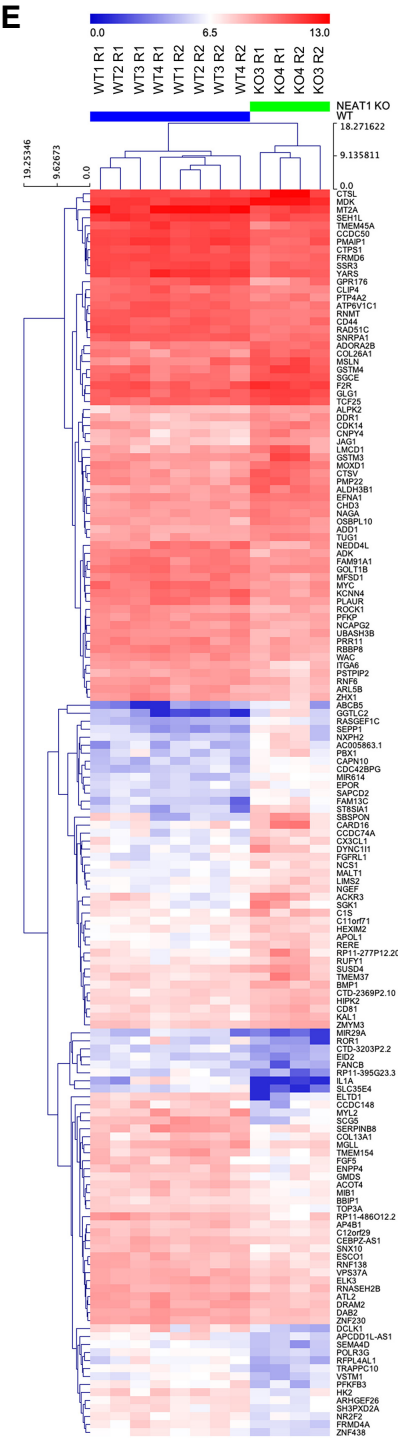

FIGURE 5. NEAT1_1 KO only causes limited changes in gene expression. (A) Representative NEAT1/NEAT1_2 FISH in $\mathrm{G}_{0}$ and $\mathrm{G}_{1} \mathrm{~S}$ conditions as in Figure 2A-C in WT and NEAT1_1 KO cells used for the Smartseq2 RNA sequencing experiment. (B) Number of genes detected in $\mathrm{G}_{0}$ and $\mathrm{G}_{1} \mathrm{~S}$ conditions. Red/pink dots are WT conditions; blue dots represent $\mathrm{KO}$ conditions. Significance was tested using a two-sided unpaired $t$-test comparing the number of genes detected in WT and $K O$ conditions. $(C, D)$ Volcano plot of gene expression changes $\left(-\log _{2}\right)$ in $G_{0}$ $(C)$ and $G_{1} S(D)$ plotted against their $P$-value $\left(-\log _{10}\right)$. Dots are color-coded red if the adjusted $P<0.05$, orange if $\log _{2}$ fold change $>1$, and green if both. (E) Hierarchical clustering of $G_{0}$ samples based on 156 unique differentially expressed genes $(F C>1.5, P$-adj<0.05). (F) Hierarchical clustering of $G_{1} / S$ samples based on 23 unique differentially expressed genes (FC>1.5, P-adj. <0.05).

nests from WT, Neat1 full KO, Neat1 heterozygous, PAS heterozygous, and PAS KO mothers at 3 and 6 wk of age and confirmed that full $\mathrm{KO}$ females were unable to successfully nurture their pups. In contrast, PAS KO females gave birth to normally sized nests, and all offspring developed and gained weight normally (Fig. 6E,F).

To further study the impact of the mutation on the growth of normal cells in vitro, we produced PAS KO MEFs. Despite an increase in Neat1_2 levels

(Supplemental Fig. S7A-C), passage 3 MEFs derived from the PAS KO mice grew similarly to WT MEFs. Full Neat $1 \mathrm{KO}$ also grew similarly to WT fibroblasts (Supplemental Fig. S7D,E; Nakagawa et al. 2011; Adriaens et al. 2016).

\section{Mouse Neat1_1 does not contribute to DNA damage induction and reduced growth during skin carcinogenesis}

The skin of Neat1 KO mice exhibits an exacerbated sensitivity to DNA damage and, thereby, an increased resistance to DMBA/TPA-induced skin hyperplasia and tumorigenesis (Adriaens et al. 2016). To test whether Neat1_1 mice exhibit a similar phenotype, we subjected these mice to the DMBA-TPA protocol and assessed PS formation and measured hyperplasia and accumulation of DNA damage in their treated back skin. We found that in our short-term protocol $111 \mathrm{~d}$ of treatment), both PAS KO and WT cells displayed moderate to severe hyperplasia (Fig. 6H) and abundant PSs (Fig. 6I). In contrast, back skin of Neat1 KO mice neither displayed PSs nor marked hyperplasia (Fig. 6H, I); in addition, it showed a significant increase in persisting DNA damage in the treated regions as compared to the skin of WT and PAS KO animals (Fig. 6J,K). We concluded that the phenotypes observed in Neat1 KO mice are attributable to the loss of the long Neat1_2 isoform and, thereby, likely to be a consequence of loss of PS nuclear bodies.

\section{DISCUSSION}

In this work, we demonstrate that the expression levels of the two NEAT1 isoforms are dynamically regulated during the cell cycle (Supplemental Fig. S8). We observed that the short isoform, NEAT1_1 is highly expressed in the $G_{0} / G_{1}$ phase of the cell cycle and that, in line with previous findings (Li et al. 2017), it localizes prominently outside of PSs. We also observed that NEAT1_1 levels drop abruptly as cells transit from the $G_{1}$ to the $S$ phase. This is consistent with the observation that NEAT1_1 is detected at high levels in terminally 
A

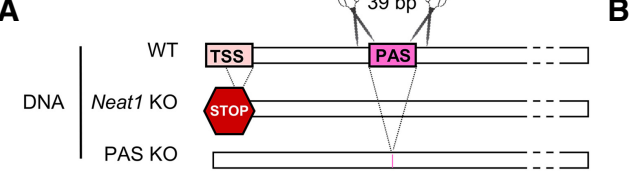

D

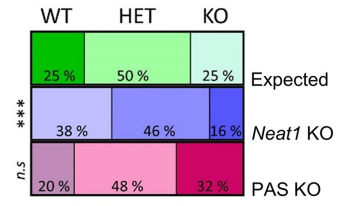

G

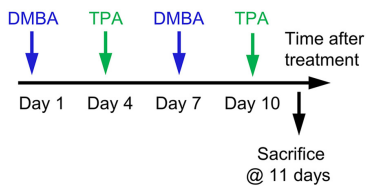

$\mathbf{H}$

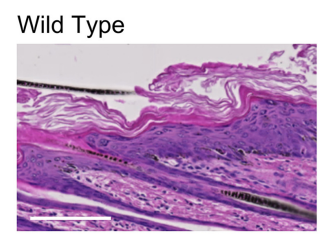

Neat1 KO

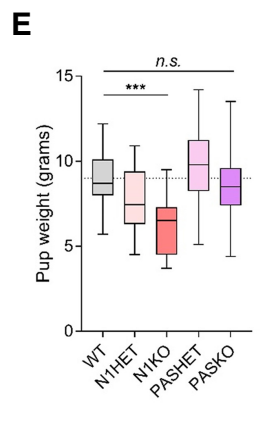

F

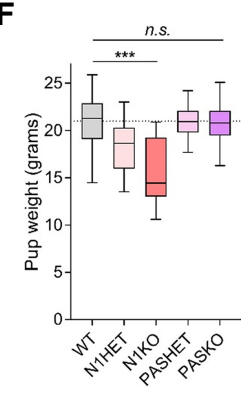

C

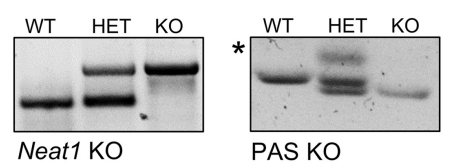

DAPI Neat1 Neat1 2 K5
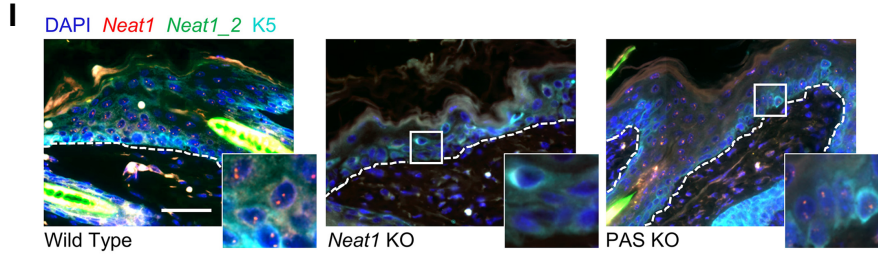

$\mathbf{J}$

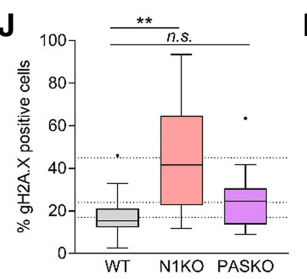

$\mathbf{K}$
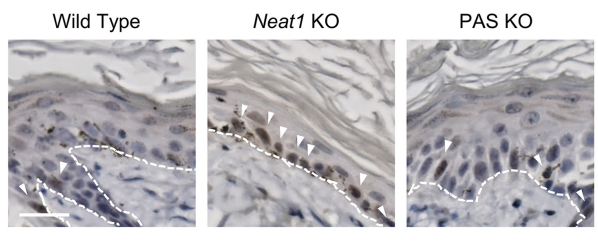

FIGURE 6. Comparison of Neat1 versus Neat1_1 KO phenotypes. (A) CRISPR strategy to knock out Neat1_1 in mouse embryonic stem cells resulting in a 39-base pair deletion spanning the Neat1_1 PAS and strategy to knock out both isoforms as described in Nakagawa et al. (2011). (B) Genotyping of Neat1 full KO mice. (C) Genotyping of Neat1_1 (PAS) KO mice. The asterisk indicates an unspecific band in the heterozygous sample. (D) Genotype distribution of pups born from heterozygous parents in full Neat1KO (middle bar) and PAS KO nests (lower bar) as compared to the expected Mendelian ratios (upper bar). $N=53$ and 15 litters for Neat 1 and PAS KO, respectively. P-values were calculated using the $\chi^{2}$ test. $\left.{ }^{* * *}\right) P<$ 0.001 . (n.s.) Not significant. $(E, F)$ Pup weight of offspring from females with the respective genotypes at $3(E)$ and $6(F)$ weeks of age. Tukey plots of pups from $N=$ between four and 19 females per genotype. Statistical significance was calculated using two-way ANOVA with Dunnett's correction for multiple testing. $\left(^{* * *}\right) P<0.001$. (n.s.) Not significant. (G) Strategy and timeline for short-term DMBA/TPA carcinogenesis protocol. (H) Representative H\&E staining of back skin sections from mice treated as in G. Scale bar, $50 \mu \mathrm{m}$. (I) Neat1 RNA-FISH and Keratin 5 immunofluorescence on back skin section of mice treated with DMBA and TPA as in G. $(J, K)$. Quantification $(J)$ and representative images $(K)$ of sections from DMBA/TPA treated back skin stained with the DNA damage marker $\gamma-\mathrm{H} 2 \mathrm{~A}$.X of $N=$ at least three mice per genotype according to the method described in Adriaens et al. (2016). The Tukey plot in J graphs individually quantified pictures. Statistical testing was done on biological replicates (averages for individual mice) using a one-way ANOVA with Sidak's correction for multiple testing. $\left.{ }^{* *}\right) P<$ 0.01 . (n.s.) Not significant. White arrows in $K$ indicate $\gamma$-H2A.X-positive cells, whereas dotted lines separate the dermis from the epidermis. Scale bar, $20 \mu \mathrm{m}$.

differentiated cells in most tissues (Nakagawa et al. 2011). In contrast, NEAT1_2 levels remain relatively constant throughout the cell cycle, and consequently NEAT1_2 is the only detected NEAT1 isoform in S phase and onward in these cells, which is in keeping with previous data reporting the presence of PSs in amitotic (interphase) cells as evidenced by the typical clustering of canonical PS protein p54nrb/NONO in their nuclei (Fox et al. 2005).

It had been proposed that the short NEAT1 isoform is recruited into PSs to support their stability and/or functions (Sasaki et al. 2009; Souquere et al. 2010; Naganuma et al. 2012; West et al. 2016). In disagreement with this possibility, we show that when both isoforms are coexpressed in $G_{0} / G_{1}$, a large fraction of the NEAT1_1 isoform localizes outside of PSs. In cells residing in other phases of the cell cycle, NEAT1_2 is the only isoform present and therefore PSs are, by and large, NEAT1_1-free in these cells. Our data is, however, in line with previous quantifications of NEAT1_1 RNA levels, indicating that, on the basis of stoichiometry, NEAT1_1 transcripts are not likely to locate to PSs or at least not in significant amounts (Chujo et al. 2017; Li et al. 2017). Our data also suggest that PSs can be assembled in the absence of NEAT1_1. This observation resonates with previous work showing that NEAT1_2 is the NEAT1 isoform required for PS assembly (Sunwoo et al. 2009), and that NEAT1_1 expression alone is not sufficient to rescue PS formation upon NEAT1_2 ablation (Sasaki et al. 2009; Naganuma et al. 2012).

Our data demonstrate that the NEAT1_1 transcript is actively degraded as cells commit to divide. We provide evidence that this process is mediated by the main RNA degradation machinery in the nucleus, namely, the RNA exosome (Vanacova and Stef 2007). Knockdown of one of its core components, RRP40, led to the specific accumulation of the NEAT1_1 short isoform within nuclear bodies 
containing persistent poly $(\mathrm{A})^{+}$RNA transcripts (Silla et al. 2018). We provide evidence that the RNA exosome mainly targets NEAT1_1 and, only to a much lesser degree, NEAT1_2. This can be explained by differences in the need for turnover between the two RNAs in their functional context. Moreover, NEAT1_1/poly(A) ${ }^{+}$-containing foci did not overlap with PSs, consistent with NEAT1_1 being spatially distinct.

Our observations that the evolutionarily conserved NEAT1_1 isoform (i) is produced at high levels in mostif not all-resting cells (as well as in cancer cells in $G_{1}$ ), and (ii) gets actively degraded as cells commit to divide suggested a putative role for NEAT1_ 1 in the regulation of the cell cycle. However, silencing of RRP40 did not overtly perturb progression of the cell cycle. Consistently, previous reports have shown that modulating levels of neither RRP40 and its targets (Graham et al. 2009; Zinder and Lima 2017) nor NEAT1_1 affect cell cycle progression (for review, see Yu et al. 2017). More strikingly, although potentially biased by our cell selection procedure after CRISPR deletion of the PAS, NEAT1_1 isoform-specific $\mathrm{KO}$ cells grew and responded to stress comparably to WT control cells. Moreover, PSs formed normally in these cells, and the phenotypes observed upon silencing of NEAT1_2 in various cancer cell lines were also observed in NEAT1_1 KO cells when co-depleted for NEAT1_2. Together, these data indicated that NEAT1_2functions independently of NEAT1_1, and that NEAT1_2 can promote PS formation in the absence of the short NEAT1 isoform.

Our data demonstrating that NEAT1_1 localizes outside of PSs are consistent with another report showing that there is a fraction of NEAT1 that localizes diffusely throughout the nucleoplasm (Li et al. 2017) and the possibility that NEAT1 binds active transcription start sites in euchromatin (West et al. 2014). However, only minor changes in gene expression patterns were observed in NEAT1_1 $\mathrm{KO}$ cells, indicating that NEAT1_1 does not directly impact on transcription. Instead, we hypothesize that its enrichment in euchromatin might have spatial, physical grounds (i.e., diffusion is easier in euchromatin because of a decrease in molecular crowding) rather than a specific functional role in modulating gene expression. Moreover, in contrast to full Neat1 KO mice (Nakagawa et al. 2014), their Neat1_1-only counterparts were born at the normal Mendelian ratios. Pups born from Neat1_1 KO females were not significantly smaller than those from WT and heterozygous mothers. This is in contrast to our previously published observation that Neat1 $\mathrm{KO}$ females cannot nurture their pups properly owing to mammary gland formation and lactation defects (Standaert et al. 2014). Similarly, Neat1_1 KO mice did not exhibit any of the phenotypes observed in Neat1 KO mice exposed to a twostep skin carcinogenesis protocol (Adriaens et al. 2016). In contrast, other groups have found that transient overexpression of NEAT1_1 could, at least partially, counter a
NEAT1 loss phenotype (e.g., in pancreatic or prostate cancer) (Chakravarty et al. 2014; Mello et al. 2017) and, thus, NEAT1_1-specific functions, in particular in the pathophysiological context, need to be further investigated.

Taken together, our data suggest that, at least in the interrogated experimental conditions and specific contexts of our study, NEAT1_1 is a nonfunctional, nonessential isoform in both resting and proliferative cells. Is NEAT1_1 then just an unimportant by-product of the NEAT1 locus? Perhaps active transcription of the NEAT1 locus ensures that cells can rapidly switch to NEAT1_2 production in response to stress and thereby the formation of PS. Constant synthesis of a nonfunctional NEAT1_1 transcript would therefore be the price that cells have to pay to be able to quickly engage a PS-dependent survival pathway when exposed to deleterious stimuli. However, it cannot be excluded that NEAT1_1 does exert a function in very specific stress and/or pathophysiological conditions, for instance during viral infections (Saha et al. 2006; Zhang et al. 2013; Imamura et al. 2014; Ma et al. 2017; Morchikh et al. 2017; Wang et al. 2017). The enclosed described Neat1_1 mouse and cellullar models will be a valuable tool to test this possibility.

\section{MATERIALS AND METHODS}

\section{Cell lines, culture methods, and cell synchronization}

U2OS, HeLa, and HCT116 WT and p53KO isogenic cell lines were obtained from the LGC ATCC and maintained in DMEM (Thermo Fisher Scientific cat. no. 21885025) plus 10\% fetal bovine serum (Fisher Scientific cat. no. 10270106). None of the cell lines used was reported in the ICLAC database of commonly misidentified cell lines. All cell lines were tested monthly for mycoplasma contamination and found negative. After their initial purchase, cell lines were not further authenticated. For synchronization in $\mathrm{G}_{0}$, the cells were washed with PBS $24 \mathrm{~h}$ after plating and media were replaced with media containing no serum for $3 \mathrm{~d}$. For $\mathrm{G}_{1}$ / $\mathrm{S}$ synchronization, $\mathrm{G}_{0}$ cells were released in $20 \%$ serum plus 5 $\mu \mathrm{g} / \mathrm{mL}$ aphidicolin (Sigma-Aldrich A0781) for $24 \mathrm{~h}$. For $\mathrm{G}_{1}, \mathrm{~S}$, and $G_{2}$ synchronization, media was replaced with media containing $2 \mathrm{mM}$ thymidine (Sigma-Aldrich T1895) for $12 \mathrm{~h}$, released in normal growth media for $12 \mathrm{~h}$, and then grown again in thymidine-containing media for $12 \mathrm{~h}$ prior to release in normal media and harvesting at the indicated time points. RNA stability experiments were performed using $5 \mu \mathrm{g} / \mathrm{mL}$ actinomycin $\mathrm{D}$ (Sigma-Aldrich A1410) or $40 \mu \mathrm{g} / \mathrm{mL} \alpha$-amanitin (Sigma-Aldrich A2263) for the indicated time points. For knockdown experiments with siPOOLs (siTOOLS Biotech), 25 nM siRNA was transfected using Lipofectamine RNAiMAX (Thermo Fisher Scientific, cat. no. 13778075) as previously described (Adriaens et al. 2016). siRNA against RRP40 and eGFP were described in Silla et al. (2018).

\section{Generation and culture of mouse embryonic fibroblasts}

Mouse embryonic fibroblasts were generated from plug-checked pregnant females at E12.5. The embryos were removed from the 
uterus, and internal organs were discarded with sterile forceps. Heads were used for genotyping. The remainder tissue was pipetted up and down in sterile PBS several times to obtain singlecell suspensions before transfer to tissue culture dishes with DMEM containing 10\% serum, 1\% penicillin/streptomycin (Invitrogen, cat. no. 15140122), and $50 \mu \mathrm{M} \beta$-mercaptoethanol (Thermo Fisher Scientific, cat. no. 31350010). The cells were passaged twice before all experiments were performed at passage 3.

\section{RNA-FISH}

Dual RNA-FISH was performed according to the Stellaris RNAFISH protocol essentially as described in Adriaens et al. (2016) but with probesets against both (cat. no. VSMF-2246-5 and VSMF-2247-5) or NEAT1_2 specifically (cat. no. VSMF-2251-5) in human cells and against Neat1 both (cat. no. VSMF-3030-5) or Neat1_2 (cat. no. VSMF-3035-5) in mouse cells and mouse tissues. According to the manufacturer's documentation, the probeset targeting both isoforms was designed against nuclear paraspeckle assembly transcript 1 (nonprotein coding) NEAT1_5 a.k.a. TncRNA; LINC00084; NCRNA00084 (NCBI gene ID:283131) and nucleotides 1-3756 of NR_028272.1, thus targeting the overlapping region of both isoforms. In contrast, the probeset targeting the long isoform was designed against the middle of a long variant of GQ859162 nucleotides 3800-11700. For the probes targeting mouse Neat1 transcripts, the probeset was designed against mouse nuclear paraspeckle assembly transcript 1 (nonprotein coding), Neat1_5, a.k.a. VINC; 2310043N10Rik (NCBI gene ID: 66961), and the short and long variant of NR_003513.2 nucleotides 1-3177. Mouse long-isoform-targeting probes were designed against mouse nuclear paraspeckle assembly transcript 1 (nonprotein coding), Neat1_m, a.k.a. VINC; 2310043N10Rik (NCBI gene ID: 66961), and the middle of the long variant of GQ859163 nucleotides 4001-12000. Further information and documentation can be found on the Stellaris website (https://www.biosearchtech.com/products/rna-fish).

Generally, cells were plated on 11-mm coverslips in six-well plates to allow for concomitant RT-qPCR analysis, cell cycle analysis, and RNA-FISH. Costaining with the paraspeckle marker p54nrb/NONO (Souquere et al. 2010) was done after the ethanol permeabilization step of the RNA-FISH protocol. Briefly, the cells were washed once with PBS followed by a $5^{\prime}$ permeabilization step with $0.5 \%$ Triton-X100 in PBS. Then, the cells were incubated with a 1/1000 dilution of the antibody in DAKO antibody dilution reagent for $1 \mathrm{~h}$ at room temperature followed by three washes in $0.05 \%$ Tween-20 in PBS and staining with the secondary antibody (Life Technologies, anti-mouse A488) in DAKO for $1 \mathrm{~h}$ at RT. After two short washes in $0.05 \%$ Tween-20 in PBS, the cells were incubated with wash buffer ( $2 \times$ SSC, 10\% v/v formamide) (Sigma-Aldrich, cat. no. F9037) and the RNA-FISH protocol was continued as described. Hybridization buffer was made using the same formula as wash buffer, adding $10 \% \mathrm{w} / \mathrm{v}$ dextran (Sigma-Aldrich, cat. no. D8606) and probes at a final concentration of $25 \mathrm{nM}$. Images were acquired with a Nikon A1 confocal microscope acquired through a Hercules grant type 1 AKUL/09/037 and processed for overlay and brightness and contrast adjustments using ImageJ. RNA-FISH images from mouse back skin tissue were acquired with a ZEISS Axio Scan Z1 microscope using
$20 \times$ and $40 \times$ objectives followed by stitching of the continuous fields using ZEN2 software.

\section{Image analysis}

Confocal images were quantified using Fiji software (ImageJ 1.51p. Java version 1.8.0_66, 64-bit, National Institutes of Health). To determine the number of cells that display NEAT1_1 outside of paraspeckle nuclear bodies, we processed the raw images with the Speckle Inspector plug-in on each channel after thresholding, with a minimal speckle size of 2 pixels, within the nuclear region delineated by the DAPI channel. The number of spots in the NEAT1_2 channel was subtracted from the number of spots per cell in the NEAT1 channel. If the outcome of the subtraction was $>3$ (arbitrary error margin: 0 [expected] +3 to account for accidental background spots in the Q570 channel), we considered that the cells contained detectable NEAT1_1 outside of the PSs. The total numbers of cells were determined using the Cell Counter plug-in. Nuclear RNA-FISH intensity (Figs. 3G, 4B) was calculated by thresholding, filling holes, and watershed of the DAPI channel, and determination of the nuclear intensity in the NEAT1 and NEAT1_2 channels per cell via the "send to" functionality in "Set Measurements" before "Counting Particles." The percentage of cells containing detectable NEAT1_1 is represented on the left $y$-axis, whereas the percentage of cells that only displayed PSs was represented on the right $y$-axis.

To find the ratios per cell of NEAT1_2 signal over total NEAT1 signal, we used the Fiji function "Set Measurements" as above to redirect DAPI-thresholded images to the respective NEAT1 (Quasar 570, measured in the red channel and represented in red) and NEAT1_2 (Quasar 670, measured in the far red channel and represented in blue) channels to obtain their relative intensities, which were then plotted per cell as NEAT1_2/NEAT1 relative integrated density per cell.

\section{RNA isolation and RT-qPCR}

RNA isolation, generation of cDNA, and RT-qPCR were performed essentially as described in Adriaens et al. (2016). Briefly, after lysis, the cell-lysis buffer mixture was heated for $10 \mathrm{~min}$ at $55^{\circ} \mathrm{C}$ according to the protocol described in Chujo et al. (2017). Then, total RNA was isolated using the NucleoSpin RNA kit (Macherey Nagel, cat. no. 740955), including rDNAse treatment for $15 \mathrm{~min}$ according to the manufacturer's instructions. The RNA was reverse-transcribed using the Thermo Fisher Scientific High Capacity cDNA Reverse Transcription Kit (cat. no. 4368813). RT-qPCR was performed with GC Biotech SensiFast SYBR No-Rox (cat. no. BIO-98020) and run on a Roche LightCycler-480-384. For normalization, the geometric mean of the two most stable reference genes out of at least three was calculated using geNorm in qBase+ Software (Biogazelle; www .qbaseplus.com). RT-qPCR primer sequences were as follows: NEAT1 fw: 5'-GGAGAGGGTTGGTTAGAGAT-3'; NEAT1 rev: 5'CCTTCAACCTGCATTTCCTA-3'; NEAT1_2 fw: 5'-GGCCAGAG CTTTGTTGCTTC-3'; NEAT1_2 rev: 5'-GGTGCGGGCACTTACT TACT-3'; CDKN1A fw: 5'-AGCAGAGGAAGACCATGTGGA-3'; CDKN1A rev: 5'-AATCTGTCATGCTGGTCTGCC-3'; UBC fw: 5'ATTTGGGTCGCGGTTCTTG-3'; UBC rev: 5'-TGCCTTGACATTC TCGATGGT-3'; TBP fw: 5'-CGGCTGTTTAACTTCGCTTC-3'; 
TBP rev: 5'- CACACGCCAAGAAACAGTGA-3'; B2M fw: 5'TGCTGTCTCCATGTTTGATGTATCT-3'; B2M rev: 5'-TCTCTG CTCCCCACCTCTAAGT-3'; HPRT1 fw: 5'-TGACACTGGCAA AACAATGCA-3'; HPRT1 rev: 5'-GGTCCTTTTCACCAGCAAGC T-3'; GAPDH fw: 5'-TGCCATGTAGACCCCTTGAAG-3'; GAPDH rev: 5'-ATGGTACATGACAAGGTGCGG-3'; $18 \mathrm{~S}$ fw: 5'-TT CGGAACTGAGGCCATG-3'; $18 \mathrm{~S}$ rev: 5'-TTTCGCTCTGGTC CGTCT-3'; mNeat1_2 fw 5'-GCTCTGGGACCTTCGTGACTCT3'; mNeat1_2 rev 5'-CTGCCTTGGCTTGGAAATGTAA-3'; mNeat1 fw 5'-TTGGGACAGTGGACGTGTGG-3'; mNeat1 rev 5'-TCAA GTGCCAGCAGACAGCA-3'; mHmbs fw 5'-GCGGA GTCATGTC CGGTAA-3'; mHmbs rev 5'-GTGGTGGACATAG CAATGATTT-3'; mGapdh fw 5'-AGGTTGTCTCCTGCGAC TTCA-3'; mGapdh rev 5'-GGTGGTCCAGGGTTTCTTACTC-3'. For correlation analysis, primer efficiencies were calculated in qBase+ by combining cDNA of each of the tested samples and producing a serial dilution $(1,0.5,0.25,0.125,0.0625,0.03125)$ to be run simultaneously with the individual samples.

\section{Cell cycle analysis}

Cell cycle analysis was performed by pulsing the cells with $10 \mu \mathrm{M}$ of EdU for 30 min before harvesting and trypsinization, or via DNA profiling alone against a nonsynchronized control to identify $2 \mathrm{~N}$ and $4 \mathrm{~N}$ populations. For EdU staining, the cells were washed in cold PBS $+10 \%$ serum to inactivate the trypsin, collected by centrifugation and fixed for 15 min with 4\% PFA in PBS. After two washes in PBS, the cells were stored overnight in $15 \mathrm{~mL} 0.01 \%$ Triton-X100 at $4^{\circ} \mathrm{C}$. To detect cells in S phase, the cells were subjected to a modified Click-IT reaction protocol (Click-iT EdU Alexa Fluor 488, cat. no. C10420). Briefly, the cells were collected by centrifugation, the supernatant was discarded, and the cells were incubated in $50 \mu \mathrm{L}$ Click-IT reaction cocktail $(43.75 \mu \mathrm{L}$ PBS + $1 \mu \mathrm{LuSO}_{4} 100 \mathrm{mM}+5 \mu \mathrm{L} 100 \mathrm{mM}$ ascorbic acid $+0.25 \mu \mathrm{L}$ A488 Azide dye) for $50 \mathrm{~min}$. The cells were then washed once in PBS and resuspended in $300 \mu \mathrm{L}$ DAPI staining buffer $(5 \mu \mathrm{g} /$ $\mathrm{mL}$ DAPI in PBS with $0.1 \% \mathrm{w} / \mathrm{v}$ BSA) before analysis on a MACSquant VYB flow cytometer (Miltenyi Biotec).

\section{CRISPR/Cas9 plasmid construction}

Guide RNAs were designed for the $3^{\prime}$ regulatory region of NEAT1_1 using http://crispr.mit.edu. Five micrograms of plasmid pSpCas9(BB)-2A-GFP (pX458) (Addgene, cat. no. 48138) was digested with Bbsl/Bpil (Thermo Fisher Scientific) and purified using a NucleoSpin Gel and PCR Clean-up Kit (Macherey Nagel, cat. no. 740609) followed by in-fusion cloning of annealed gRNA oligos with 20-nt overhangs on both sides (IDT) with sequences according to the manufacturer's instructions (Takara Bio cat. no. 121416). The gRNA sequences used to generate four different Cas9 targeting plasmids were upstream Guide \#1 5'-GTGTATTAGTCACGCATGTATGG-3' quality score 89; upstream Guide \#7 5'-GTACTGGTATGTTGCTCTGTATGG-3', quality score 70; downstream Guide \#1 5'-GTACATCCAAAGTC GTTATGAAGG-3', quality score 90; downstream Guide \#4 5'GCGTTATGAAGGCAATGTGATAGG-3', quality score 70 . Following in-fusion cloning, the plasmids were transformed into competent bacteria ( $\mathrm{DH} 5 \alpha)$ grown on ampicillin plates. A colony PCR was performed to check for the correct insertion of the gRNA sequence using GoTaq Green Mastermix (Promega, cat. no. M712) and primers 5'-GAGGGCCTATTTCCCATGATT-3' (fw) and 5'-AAAAAAGCACCGACTCGGTGCCA-3' (rev). Positive clones were further expanded and their inserted sequences were verified with Sanger sequencing at the VIB Genomic Service Facility, Belgium using the same primers.

\section{Generation of NEAT1_1 KO cells}

Once we obtained the desired Cas9/gRNA constructs, we transfected cells plated in 10-cm dishes with $10 \mu \mathrm{g}$ of downstream and $10 \mu \mathrm{g}$ of upstream plasmid (Combination dG1/uG1 for U2OS and HCT116 and dG4/uG7 for HCT116) using a standard transient overexpression protocol with Lipofectamine 2000 reagent according to the manufacturer's instructions (Thermo Fisher Scientific, cat. no. 11668019). Forty-eight hours after transfection, we sorted the cells for GFP expression using a S3 Sorter (Bio-Rad Laboratories) and diluted the cells at 0.5 cells $/ 100 \mu \mathrm{L}$ into 96-well plates. After 2 wk of culture, we visually inspected the wells and selected those containing a single clone. These were collected and replated in duplicate. The cells in one of the two wells were then lysed and subjected to PCR analysis to determine their NEAT1_1 genotype with primers $5^{\prime}$-CGTTGGGAT CTTTCTGTCT-3' (fw) and 5'-GCTCTCCTACATGGCCTTAAT-3' (rev). These primers were also used for Sanger sequencing to characterize the repair on each allele in homozygous NEAT1_1 KO clones. Several homozygous WT and homozygous KO clones were then selected and expanded into new cell lines from the remaining wells.

\section{Cell growth assays}

To determine long-term cell growth, cells were plated at the indicated densities in three wells per cell line per experiment and grown for 10 or $14 \mathrm{~d}$. They were washed twice in cold PBS, followed by staining for 15 min with $0.5 \%$ Crystal Violet (SigmaAldrich, cat. no. C6158) in $20 \%$ methanol/ $80 \% \mathrm{H}_{2} \mathrm{O}$. The plates were washed and rinsed in tap water and the percentage of area covered of the wells was quantified using Fiji. For shortterm growth assays, 1500 cells were plated followed by incubation with WST-1 reagent (Roche, cat. no. 05015944 001) and measurement of the luminescence with a VICTOR X3 Multilabel Plate Reader (PerkinElmer) at the indicated time points. Cells were treated with $10 \mu \mathrm{M}$ Nutlin-3a (Sigma-Aldrich, cat. no. SML05080) or $150 \mathrm{ng} / \mathrm{mL}$ doxorubicin (Sigma-Aldrich, cat. no. D1515).

\section{RNA sequencing}

Total RNA was extracted as described above using the NucleoSpin RNA kit (Macherey Nagel, cat. no. 740955). The RNA integrity was monitored using Bioanalyzer analysis (Agilent, RIN: 9.7-10). About 500 pg of RNA per sample was reversetranscribed and amplified using a modified SMARTseq2 protocol (Rambow et al. 2018). Prior to generating sequencing libraries using the NexteraXT kit (Illumina, cat. no. FC-131-10), cDNA profiles were monitored using the Bioanalyzer. Sequencing was performed on a Nextseq500 platform (Illumina, SE75bp). Reads 
were then mapped to the human genome (hg19) using STAR (2.4.1b) and quantified with Subread (1.4.6-p2). Differential analyses between NEAT1_1 $\mathrm{KO}$ and WT samples (during $\mathrm{G}_{0}$ and $\mathrm{G}_{1} / \mathrm{S}$ ) were executed using the DeSeq2 pipeline. Samples were grouped using hierarchical clustering (Euclidean distance) based on differentially expressed genes (MeV4_8_1). Sequencing data was deposited in the Gene Expression Omnibus (GEO) (https://www.ncbi.nlm.nih.gov/geo/) under accession number GSE137211.

\section{KO mice}

Neat1 KO, Neat1_1 KO, and WT mice were maintained on a pure C57BL/6J background in a certified animal facility at KU Leuven Campus Gasthuisberg, Leuven, Belgium. They were maintained on a 12/12 h light-dark cycle and had access to food and water ad libitum. All animal experiments were carried out in accordance with the guidelines of the Ethical Committee University of Leuven Animal Care and Use under project license 089/2013. Full Neat1 KO mice were described previously (Nakagawa et al. 2011) and genotyped with primers 5'-GGTGACGCGACACAAGAGTA-3' (fw), 5'-AAATGTGAGCGAGTAACAACCC-3' (rev WT) and 5'CTGTGAAACTTGTGCCCTCC-3' (rev KO), giving rise to PCR products of $612 \mathrm{bp}$ (Neat1 KO) and $336 \mathrm{bp}$ (WT). Neat1_1 KO mice were generated by S. Nakagawa and T. Hirose using a similar CRISPR/Cas9 strategy as described for the cancer cells above generating a 39-bp deletion of the PAS (5'-ACAGCAAAATA AAGGTTTGAGATTGAAGCTTCTTAGAAT-3') and genotyped with primers 5'-GCAAAGT GACAGAGGTCGAGA-3' (fw) and 5'AGGCAAAGTGACAGAGGTCG-3' (rev) (WT allele: 145 bp; KO allele, 106 bp) (Isobe et al. 2019). To test for lactation defects, mice with mothers from the indicated genotypes were weighed at 3 and 6 wk of age (Standaert et al. 2014). Ratios of animals born at indicated genotypes to test against expected Mendelian genotype ratios were calculated from heterozygous $\times$ heterozygous parents in both colonies.

\section{DMBA/TPA protocol}

The DMBA/TPA protocol was performed as described in Adriaens et al. (2016).

\section{H\&E and immunohistochemistry}

Immunohistochemistry and quantification of images were performed as described in Adriaens et al. (2016) using antibodies against $\gamma$-H2A.X (Cell Signaling 2577; 1/1400) and keratin 5 (rabbit polyclonal anti-keratin 5; Covance, PRB-160P-0100; 1/1000). For immunofluorescence the secondary antibody was antiRabbit-A488 (Life Technologies). Images were acquired with a ZEISS Axio Scan Z1 microscope using $20 \times$ and $40 \times$ objectives followed by stitching of the continuous fields using ZEN 2 software.

\section{SUPPLEMENTAL MATERIAL}

Supplemental material is available for this article.

\section{COMPETING INTEREST STATEMENT}

J.-C.M. owns a patent to target NEAT1_2 in cancer (US9783803B2) and is a cofounder of NewCo, a company aiming to develop oligo-based therapeutics to target cancer. The other authors declare no competing interests.

\section{ACKNOWLEDGMENTS}

We thank Odessa Van Goethem and Veronique Benne for excellent technical support and maintenance of the mouse colonies. We thank Tom Misteli ( $\mathrm{NCl} / \mathrm{NIH}$, USA), Michael Dewaele (VIB, Belgium), Paulo P. Amaral (Cambridge University, UK), and Andrew Blackford (Oxford University, UK) for helpful discussions throughout the project. C.A. was supported by an IWT fellowship (no. 141372) from the Belgian Research Foundation-Flanders (FWO) and a Gustave Boël-Sofina grant (V433017N) from the Koning Boudewijn Stichting, Belgium. The work in the J.-C.M. laboratory was supported by KUL GOA (\#14/012), VLK (Vlaamse Liga tegen Kanker), Interreg (SKiN-HUID), and Fonds voor Wetenschappelijk Onderzoek Vlaanderen (\#G.0929.16N). This work was supported by the Japan Society for the Promotion of Science (JSPS) KAKENHI grant number $17 \mathrm{H03604}$ and Ministry of Education, Culture, Sports, Science and Technology (MEXT) KAKENHI grant number 26113005 (S.N.) We thank the members of the Research Resource Center at the RIKEN Brain Science Institute for the generation of the PAS KO mice. Work in the T. H.J. laboratory was supported by the European Research Council (ERC, grant 339953). We thank Manfred Schmid for computational support.

Author contributions: C.A. designed the study, performed all experiments, and analyzed all data apart from the RNA sequencing analysis. F.R. performed RNA sequencing analysis. G.B. performed experiments. T.S. provided images from RRP40 KD conditions and found the link with NEAT1_1-specific degradation. T.H., M.M., T.C., A.H., and S.N. provided reagents and made the mouse models. J.-C.M. and T.J.H. designed and supervised the study. C.A. and J.-C.M. wrote the manuscript with input from all authors.

Received March 28, 2019; accepted August 30, 2019.

\section{REFERENCES}

Adriaens C, Standaert L, Barra J, Latil M, Verfaillie A, Kalev P, Boeckx B, Wijnhoven PW, Radaelli E, Vermi W, et al. 2016. p53 induces formation of NEAT1 IncRNA-containing paraspeckles that modulate replication stress response and chemosensitivity. Nat Med 22: 861-868. doi:10.1038/nm.4135

Ahmed ASI, Dong K, Liu J, Wen T, Yu L, Xu F, Kang X, Osman I, Hu G, Bunting KM, et al. 2018. Long noncoding RNA NEAT1 (nuclear paraspeckle assembly transcript 1) is critical for phenotypic switching of vascular smooth muscle cells. Proc Natl Acad Sci 115: E8660-E8667. doi:10.1073/pnas.1803725115

Blume CJ, Hotz-Wagenblatt A, Hüllein J, Sellner L, Jethwa A, Stolz T, Slabicki M, Lee K, Sharathchandra A, Benner A, et al. 2015. P53dependent non-coding RNA networks in chronic lymphocytic leukemia. Leukemia 29: 2015-2023. doi:10.1038/leu.2015.119

Chakravarty D, Sboner A, Nair SS, Giannopoulou E, Li R, Hennig S, Mosquera JM, Pauwels J, Park K, Kossai M, et al. 2014. The 
oestrogen receptor $\alpha$-regulated IncRNA NEAT1 is a critical modulator of prostate cancer. Nat Commun 5: 5383. doi:10.1038/ ncomms6383

Chen L-L, Carmichael GG. 2009. Altered nuclear retention of mRNAs containing inverted repeats in human embryonic stem cells: functional role of a nuclear noncoding RNA. Mol Cell 35: 467-478. doi:10.1016/j.molcel.2009.06.027

Choudhry H, Albukhari A, Morotti M, Haider S, Moralli D, Smythies J, Schödel J, Green CM, Camps C, Buffa F, et al. 2014. Tumor hyp-

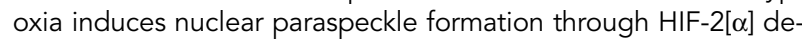
pendent transcriptional activation of NEAT1 leading to cancer cell survival. Oncogene 34: 4482-4490. doi:10.1038/onc.2014 .378

Chujo T, Yamazaki T, Kawaguchi T, Kurosaka S, Takumi T, Nakagawa S, Hirose T. 2017. Unusual semi-extractability as a hallmark of nuclear body-associated architectural noncoding RNAs. EMBO J 36: 1447-1462. doi:10.15252/embj.201695848

Clemson CM, Hutchinson JN, Sara SA, Ensminger AW, Fox AH, Chess A, Lawrence JB. 2009. An architectural role for a nuclear noncoding RNA: nEAT1 RNA is essential for the structure of paraspeckles. Mol Cell 33: 717-726. doi:10.1016/j.molcel.2009.01 .026

Fox AH, Bond CS, Lamond Al. 2005. P54nrb forms a heterodimer with PSP1 that localizes to paraspeckles in an RNA-dependent manner. Mol Biol Cell 16: 5304-5315. doi:10.1091/mbc.E05

Fox AH, Nakagawa S, Hirose T, Bond CS. 2018. Paraspeckles: where long noncoding RNA meets phase separation. Trends Biochem Sci 43: 124-135. doi:10.1016/j.tibs.2017.12.001

Graham AC, Kiss DL, Andrulis ED. 2009. Core exosome-independent roles for Rrp6 in cell cycle progression. Mol Biol Cell 20: 2242 2253. doi:10.1091/mbc.e08-08-0825

Hirose T, Virnicchi G, Tanigawa A, Naganuma T, Li R, Kimura H, Yokoi T, Nakagawa S, Benard M, Fox AH. 2014. NEAT1 long noncoding RNA regulates transcription via protein sequestration within subnuclear bodies. Mol Biol Cell 25: 169-183. doi:10.1091/mbc .E13-09-0558

Hu SB, Xiang JF, Li X, Xu Y, Xue W, Huang M, Wong CC, Sagum CA, Bedford MT, Yang $L$, et al. 2015. Protein arginine methyltransferase CARM1 attenuates the paraspeckle mediated nuclear retention of mRNAs containing IRAlus. Genes Dev 29: 630-645. doi:10.1101/gad.257048.114

Hupalowska A, Jedrusik A, Zhu M, Bedford MT, Glover DM, ZernickaGoetz M. 2018. CARM1 and paraspeckles regulate pre-implantation mouse embryo development. Cell 175: 1902-1916.e13. doi:10.1016/j.cell.2018.11.027

Idogawa M, Ohashi T, Sasaki Y, Nakase H, Tokino T. 2017. Long noncoding RNA NEAT1 is a transcriptional target of p53 and modulates p53-induced transactivation and tumor-suppressor function. Int J Cancer 140: 2785-2791. doi:10.1002/ijc.30689.

Imamura K, Imamachi N, Akizuki G, Kumakura M, Kawaguchi A, Nagata K, Kato A, Kawaguchi Y, Sato H, Yoneda M, et al. 2014. Long noncoding RNA NEAT1-dependent SFPQ relocation from promoter region to paraspeckle mediates IL8 expression upon immune stimuli. Mol Cell 53: 393-406. doi:10.1016/j.molcel.2014 01.009

Isobe M, Toya H, Mito M, Chiba T, Asahara H, Hirose T, Nakagawa S. 2019. Forced isoform switching of Neat1_1 to Neat1_2 leads to the hyperformation of paraspeckles but does not affect the development and growth of mice. bioRxiv 698068. doi:10.1101/698068

Jiang L, Shao C, Wu OJ, Chen G, Zhou J, Yang B, Li H, Gou LT, Zhang Y, Wang Y, et al. 2017. NEAT1 scaffolds RNA-binding proteins and the microprocessor to globally enhance pri-miRNA processing. Nat Struct Mol Biol 24: 816-824. doi:10.1038/nsmb.3455

Kawaguchi T, Tanigawa A, Naganuma T, Ohkawa Y, Souquere S, Pierron G, Hirose T. 2015. SWI/SNF chromatin-remodeling com- plexes function in noncoding RNA-dependent assembly of nuclear bodies. Proc Natl Acad Sci 112: 4304-4309. doi:10.1073/pnas .1423819112

Lellahi SM, Rosenlund IA, Hedberg A, Kiær LT, Mikkola I, Knutsen E, Perander M. 2018. The long non-coding RNA NEAT1 and nuclear paraspeckles are upregulated by the transcription factor HSF1 in the heat shock response. J Biol Chem 293: 18965-18976. doi:10.1074/jbc.RA118.004473

Li R, Harvey AR, Hodgetts SI, Fox AH. 2017. Functional dissection of NEAT1 using genome editing reveals substantial localization of the NEAT1_1 isoform outside paraspeckles. RNA 23: 872-881. doi:10.1261/rna.059477.116

Li S, Li J, Chen C, Zhang R, Wang K. 2018. Pan-cancer analysis of long non-coding RNA NEAT1 in various cancers. Genes Dis 5: 27-35. doi:10.1016/j.gendis.2017.11.003

Ma H, Han P, Ye W, Chen H, Zheng X, Cheng L, Zhang L, Yu L, Wu X, $X u Z$, et al. 2017. The long noncoding RNA NEAT1 exerts antihantaviral effects by acting as positive feedback for RIG-I signaling. $J$ Virol 91: e02250-16. doi:10.1128/JVI.02250-16

Mao YS, Sunwoo H, Zhang B, Spector DL. 2011. Direct visualization of the co-transcriptional assembly of a nuclear body by noncoding RNAs. Nat Cell Biol 13: 95-101. doi:10.1038/ncb2140

Mello SS, Sinow C, Raj N, Mazur PK, Bieging-Rolett K, Broz DK, Imam JFC, Vogel H, Wood LD, Sage J, et al. 2017. Neat1 is a p53-inducible lincRNA essential for transformation suppression. Genes Dev 31: 1095-1108. doi:10.1101/gad.284661.116

Modic M, Grosch M, Rot G, Schirge S, Lepko T, Yamazaki T, Lee FCY, Rusha E, Shaposhnikov D, Palo M, et al. 2019. Cross-regulation between TDP-43 and paraspeckles promotes pluripotency-differentiation transition. Mol Cell 74: 951-965.e13. doi:10.1016/j .molcel.2019.03.041

Morchikh M, Cribier A, Raffel R, Amraoui S, Cau J, Severac D, Dubois E, Schwartz O, Bennasser Y, Benkirane M. 2017. HEXIM1 and NEAT1 long non-coding RNA form a multi-subunit complex that regulates DNA-mediated innate immune response. Mol Cell 67: 387-399. doi:10.1016/j.molcel.2017.06.020

Naganuma T, Nakagawa S, Tanigawa A, Sasaki YF, Goshima N, Hirose T. 2012. Alternative $3^{\prime}$-end processing of long noncoding RNA initiates construction of nuclear paraspeckles. EMBO J 31: 4020-4034. doi:10.1038/emboj.2012.251

Nakagawa S, Naganuma T, Shioi G, Hirose T. 2011. Paraspeckles are subpopulation-specific nuclear bodies that are not essential in mice. J Cell Biol 193: 31-39. doi:10.1083/jcb.201011110

Nakagawa S, Shimada M, Yanaka K, Mito M, Arai T, Takahashi E, Fujita Y, Fujimori T, Standaert L, Marine JC, et al. 2014. The IncRNA Neat1 is required for corpus luteum formation and the establishment of pregnancy in a subpopulation of mice. Development 141: 4618-4627. doi:10.1242/dev.110544

Prasanth KV, Prasanth SG, Xuan Z, Hearn S, Freier SM, Bennett CF, Zhang MQ, Spector DL. 2005. Regulating gene expression through RNA nuclear retention. Cell 123: 249-263. doi:10.1016/ j.cell.2005.08.033

Quinn JJ, Chang HY. 2016. Unique features of long non-coding RNA biogenesis and function. Nat Rev Genet 17: 47-62. doi:10.1038/ nrg. 2015.10

Rambow F, Rogiers A, Marin-Bejar O, Aibar S, Femel J, Dewaele M, Karras P, Brown D, Chang YH, Debiec-Rychter M, et al. 2018. Toward minimal residual disease-directed therapy in melanoma. Cell 174: 843-855.e19. doi:10.1016/j.cell.2018.06.025

Saha S, Murthy S, Rangarajan PN. 2006. Identification and characterization of a virus-inducible non-coding RNA in mouse brain. J Gen Virol 87: 1991-1995. doi:10.1099/vir.0.81768-0

Sasaki YTF, Ideue T, Sano M, Mituyama T, Hirose T. 2009. MEN $\varepsilon / \beta$ noncoding RNAs are essential for structural integrity of nuclear 
paraspeckles. Proc Natl Acad Sci 106: 2525-2530. doi:10.1073/ pnas.0807899106

Shen H, Moran DM, Maki CG. 2008. Transient Nutlin-3a treatment promotes endoreduplication and the generation of therapy-resistant tetraploid cells. Cancer Res 68: 8260-8268. doi:10.1158/ 0008-5472.CAN-08-1901

Shuaib M, Parsi KM, Kawaji H, Thimma M, Adroub SA, Fort A, Ghosheh Y, Yamazaki T, Mannen T, Seridi L, et al. 2019. AGO1 in association with NEAT1 IncRNA contributes to nuclear and 3D chromatin architecture in human cells. bioRxiv 525527. doi:10.1101/525527

Silla T, Karadoulama E, Makosa D, Lubas M, Jensen TH. 2018. The RNA exosome adaptor ZFC3H1 functionally competes with nuclear export activity to retain target transcripts. Cell Rep 23: 21992210. doi:10.1016/j.celrep.2018.04.061

Singh A, Xu YJ. 2016. The cell killing mechanisms of hydroxyurea. Genes (Basel) 7: 99. doi:10.3390/genes7110099

Souquere S, Beauclair G, Harper F, Fox A. 2010. Highly ordered spatial organization of the structural long noncoding NEAT1 RNAs within paraspeckle nuclear bodies. Mol Biol Cell 21: 4020-4027. doi:10.1091/mbc.E10

Standaert L, Adriaens C, Radaelli E, Van Keymeulen A, Blanpain C, Hirose T, Nakagawa S, Marine JC. 2014. The long noncoding RNA Neat1 is required for mammary gland development and lactation. RNA 20: 1844-1849. doi:10.1261/rna.047332.114

Sunwoo H, Dinger ME, Wilusz JE, Amaral PP, Mattick JS, Spector DL. 2009. MEN $\varepsilon / \beta$ nuclear-retained non-coding RNAs are up-regulated upon muscle differentiation and are essential components of paraspeckles. Genome Res 19: 347-359. doi:10.1101/gr.087775 .108.gested

Torres M, Becquet D, Blanchard MP, Guillen S, Boyer B, Moreno M, Franc JL, François-Bellan AM. 2016. Circadian RNA expression elicited by $3^{\prime}$-UTR IRAlu-paraspeckle associated elements. Elife 5: e14837. doi:10.7554/eLife.14837
Vanacova S, Stef R. 2007. The exosome and RNA quality control in the nucleus. EMBO Rep 8: 651-657. doi:10.1038/sj.embor.7401005

Wang Z, Fan P, Zhao Y, Zhang S, Lu J, Xie W, Jiang Y, Lei F, Xu N, Zhang Y. 2017. NEAT1 modulates herpes simplex virus-1 replication by regulating viral gene transcription. Cell Mol Life Sci 74: 1117-1131. doi:10.1007/s00018-016-2398-4

Wang Y, Hu SB, Wang MR, Yao RW, Wu D, Yang L, Chen LL. 2018. Genome-wide screening of NEAT1 regulators reveals cross-regulation between paraspeckles and mitochondria. Nat Cell Biol 20: 1145-1158. doi:10.1038/s41556-018-0204-2

West JAA, Davis CPP, Sunwoo H, Simon MD, Sadreyev Rl, Wang PI, Tolstorukov MY, Kingston RE. 2014. The long noncoding RNAs NEAT1 and MALAT1 bind active chromatin sites. Mol Cell 55: 791-802. doi:10.1016/j.molcel.2014.07.012

West JA, Mito M, Kurosaka S, Takumi T, Tanegashima C, Chujo T, Yanaka K, Kingston RE, Hirose T, Bond C, et al. 2016. Structural, super-resolution microscopy analysis of paraspeckle nuclear body organization. J Cell Biol 214: 817-830. doi:10.1083/jcb .201601071

Yamazaki T, Souquere S, Chujo T, Kobelke S, Chong YS, Fox AH, Bond CS, Nakagawa S, Pierron G, Hirose T. 2018. Functional domains of NEAT1 architectural IncRNA induce paraspeckle assembly through phase separation. Mol Cell 70: 1038-1053.e7. doi:10.1016/j.molcel.2018.05.019

Yu X, Li Z, Zheng H, Chan MT, Wu WK. 2017. NEAT1: a novel cancerrelated long non-coding RNA. Cell Prolif50: e12329. doi:10.1111/ cpr.12329

Zhang Q, Chen C, Yedavalli VS, Jeang KT. 2013. NEAT1 long noncoding RNA and paraspeckle bodies modulate HIV-1 posttranscriptional expression. Am Soc Microbiol 4: e00596-12. doi:10.1128/ mBio.00596-12.Editor

Zinder JC, Lima CD. 2017. Targeting RNA for processing or destruction by the eukaryotic RNA exosome and its cofactors. Genes Dev 31: 88-100. doi:10.1101/gad.294769.116 

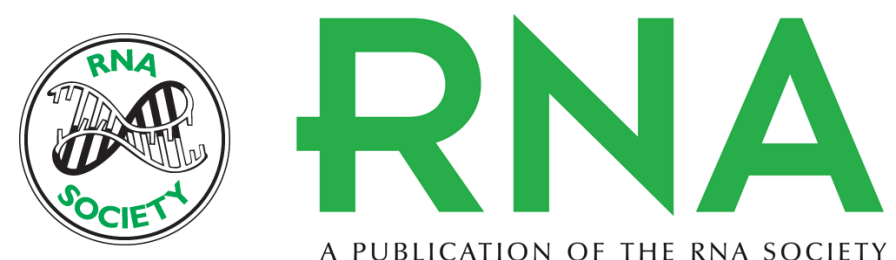

A PUBLICATION OF THE RNA SOCIETY

\section{The long noncoding RNA NEAT1_1 is seemingly dispensable for normal tissue homeostasis and cancer cell growth}

Carmen Adriaens, Florian Rambow, Greet Bervoets, et al.

RNA 2019 25: 1681-1695 originally published online September 24, 2019

Access the most recent version at doi:10.1261/rna.071456.119

\section{Supplemental http://rnajournal.cshlp.org/content/suppl/2019/09/24/rna.071456.119.DC1 \\ Material}

References This article cites 52 articles, 21 of which can be accessed free at: http://rnajournal.cshlp.org/content/25/12/1681.full.html\#ref-list-1

Creative This article is distributed exclusively by the RNA Society for the first 12 months after the Commons

License full-issue publication date (see http://rnajournal.cshlp.org/site/misc/terms.xhtml). After 12 months, it is available under a Creative Commons License (Attribution-NonCommercial 4.0 International), as described at http://creativecommons.org/licenses/by-nc/4.0/.
Email Alerting Receive free email alerts when new articles cite this article - sign up in the box at the Service top right corner of the article or click here.

\section{|||||||| Providing Precise Solutions for your research.}

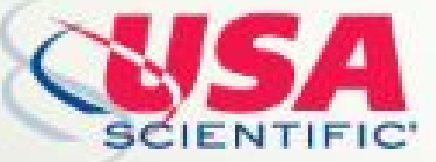

To subscribe to $R N A$ go to:

http://rnajournal.cshlp.org/subscriptions 